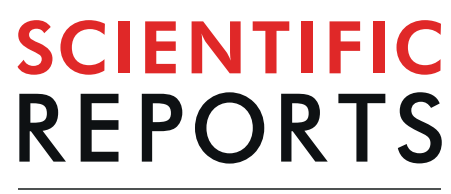

\title{
SMN complex member Gemin3 self-interacts and has a functional relationship with ALS-linked proteins TDP-43, FUS and Sod1
}

\author{
Rebecca Cacciottolo ${ }^{1,2,3}$, Joanna Ciantar ${ }^{2,3}$, Maia Lanfranco $0^{1,2,3}$, Rebecca M. Borg ${ }^{1,2,3}$, \\ Neville Vassallo ${ }^{2,3}$, Rémy Bordonné ${ }^{1}$ \& Ruben J. Cauchi ${ }^{2,3^{2}}{ }^{*}$
}

The predominant motor neuron disease in infants and adults is spinal muscular atrophy (SMA) and amyotrophic lateral sclerosis (ALS), respectively. SMA is caused by insufficient levels of the Survival Motor Neuron (SMN) protein, which operates as part of the multiprotein SMN complex that includes the DEAD-box RNA helicase Gemin3/DDX20/DP103. C9orf72, SOD1, TDP-43 and FUS are ranked as the four major genes causing familial ALS. Accumulating evidence has revealed a surprising molecular overlap between SMA and ALS. Here, we ask the question of whether Drosophila can also be exploited to study shared pathogenic pathways. Focusing on motor behaviour, muscle mass and survival, we show that disruption of either TBPH/TDP-43 or Caz/FUS enhance defects associated with Gemin3 loss-of-function. Gemin3-associated neuromuscular junction overgrowth was however suppressed. Sod1 depletion had a modifying effect in late adulthood. We also show that Gemin 3 self-interacts and $G e m 3^{\Delta N}$, a helicase domain deletion mutant, retains the ability to interact with its wild-type counterpart. Importantly, mutant:wild-type dimers are favoured more than wild-type:wild-type dimers. In addition to reinforcing the link between SMA and ALS, further exploration of mechanistic overlaps is now possible in a genetically tractable model organism. Notably, Gemin3 can be elevated to a candidate for modifying motor neuron degeneration.

Motor neuron disease (MND) encompasses a seemingly heterogeneous group of neurological conditions that are nonetheless characterised by muscle weakness and paralysis thought to arise from the selective degeneration of motor neurons. Genetic factors play a major role in disease pathogenesis and the knowledge that mutations in genes encoding RNA-binding proteins (RBPs) can lead to MND, underscores RNA dysregulation as a key contributor to motor dysfunction ${ }^{1-4}$. In infants, the predominant MND is spinal muscular atrophy (SMA), typically an autosomal recessive condition caused by inactivating mutations in the survival motor neuron 1 (SMN1) gene that are partly counteracted by the paralogous SMN2 gene. Rather than total loss of the SMN1- or SMN2-encoded SMN protein, SMA is the result of insufficient SMN levels ${ }^{5}$. SMN, operating as part of a large multiprotein complex that includes Gemins 2-8 and Unrip, is indispensable for chaperoning the assembly of spliceosomal small nuclear ribonucleoproteins ( $\mathrm{snRNPs})^{6,7}$, in addition to a possible role in the assembly and axonal trafficking of messenger ribonucleoproteins (mRNPs) in motor neurons ${ }^{8}$. In adults, the most common MND is amyotrophic lateral sclerosis (ALS), which can be inherited ( 10\%) but is mostly sporadic ( $90 \%)$. Chromosome 9 open reading frame 72 (C9orf72), Cu/Zn superoxide dismutase 1 (SOD1), transactive response DNA binding protein (TARDBP) and fused in sarcoma (FUS), in that order, are ranked as the four most common genes causing familial ALS and mutations in these genes are increasingly detected in sporadic cases ${ }^{9,10}$. TAR DNA binding-protein 43 or TDP-43 (encoded by TARDBP), and FUS are RBPs that are involved in multiple levels of RNA processing ${ }^{11,12}$.

Although SMA and ALS are traditionally considered as separate MNDs, a notion supported by differences in genetic aetiology, disease onset and type of affected motor neurons, accumulating evidence has revealed a surprising overlap at a molecular level. First, SMN and/or SMN complex members are components of the interactomes

${ }^{1}$ Institut de Génétique Moléculaire de Montpellier, CNRS-UMR 5535, Université de Montpellier, Montpellier, France. ${ }^{2}$ Department of Physiology and Biochemistry, Faculty of Medicine and Surgery, University of Malta, Msida, Malta. ${ }^{3}$ Centre for Molecular Medicine and Biobanking, Biomedical Sciences Building, University of Malta, Msida, Malta. *email: ruben.cauchi@um.edu.mt 
of SOD $1^{13}$, TDP-43 $3^{14,15}$, FUS ${ }^{16-19}$ or the dipeptide repeat (DPR) proteins resulting from hexanucleotide repeat expansion in the C9orf72 gene ${ }^{20}$. In addition, both TDP-43 and FUS were reported to localise to gems ${ }^{14,15}$, which

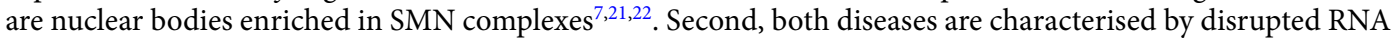
processing including snRNP perturbation ${ }^{6,15,18,23-27}$ and axonal transport defects ${ }^{8,17,28-30}$. Third, both ALS and SMA were found to co-occur within families ${ }^{31}$. Fourth, an abnormal change in SMN1 copy number including gene deletion or duplication increases susceptibility to sporadic ALS $^{32-34}$, presumably because deviations from normal SMN protein levels render motor neurons more vulnerable to degeneration. In corroboration, SMN deficiency was found to accelerate phenotypic severity in mutant SOD1 mice ${ }^{13}$. Fifth, and most important, a depleted number of gems resulting from SMN reduction was identified as a signature feature of ALS in addition to SMA ${ }^{35}$. A follow-up study unexpectedly showed that motor neurons derived from SMA or ALS patients have heterogeneous SMN levels with those having low levels being highly susceptible to cell death ${ }^{36}$. This observation explains why increasing SMN levels was found to be beneficial not only to SMA ${ }^{37,38}$ but also to ALS, at least, in SOD ${ }^{39,40}$ and TDP- $43^{41}$ mouse models. Whether SMA therapeutics elevating SMN levels are also effective in ALS patients still remains to be determined.

Known and unknown components of molecular pathways can be uncovered in an unbiased fashion via genetic approaches. Drosophila has emerged as a premier model system for this task in view of its genetic tractability ${ }^{42,43}$. Indeed, genome-wide screens in Drosophila have yielded several modifier genes that are relevant to the pathology underlying either $\mathrm{SMA}^{44-47}$ or $\mathrm{ALS}^{48-52}$. However, the overlap has been surprisingly minimal and one study even reported that overexpression or RNAi-mediated knockdown of SMN failed to modify human FUS (hFUS)-induced neurodegeneration in Drosophila eyes ${ }^{23}$. This is in contrast to earlier findings in a cell-based system showing that overexpression of SMN rescued axonal defects induced by mutant FUS ${ }^{17}$. Although a common pathway uniting SMA and ALS could have developed later in evolution, it is highly likely that the screenable phenotype used in Drosophila-based investigations was not adequate to uncover interactions between SMA- and ALS-linked proteins. Therefore, the question of whether Drosophila can be exploited to study the shared pathogenic pathway linking SMA and ALS remains. Here, we address this question by using a different approach. First, instead of SMN, we focus on Gemin3, which is a core member of the SMN complex ${ }^{7}$. Our rationale is based on accumulating evidence that has essentially shifted the limelight from SMN to its Gemin associates revealing, a previously undisclosed, starring role in the operations of the SMN complex ${ }^{6,53}$. Second, we probe for a modifying effect in muscle, a tissue that is increasingly considered as a primary site of pathogenesis in both SMA and ALS $^{54-59}$.

Gemin3, also known as DDX20 or DP103, is a DEAD-box RNA helicase which is involved in multiple cellular processes $^{6}$. Most documented are its roles in RNA metabolism, including snRNP biogenesis where it functions within the SMN complex. To this end, we have recently shown that, in Drosophila, Gemin3 interacts both genetically and physically with pICln and Tgs1, two fundamental players in the snRNP biogenesis cycle ${ }^{61}$. Here, we extend the functional relationship to three key proteins linked to ALS. Hence, we demonstrate that a combination of Gemin 3 and TDP-43 or FUS disruption exacerbates viability defects, motor dysfunction and muscle atrophy whilst suppressing neuromuscular junction (NMJ) overgrowth. Loss of Sod1 function is also responsible for inducing a prominent motoric decline in Gemin 3 mutant flies at a late stage in adult life. The likely explanation is an interference in a common pathway. Additionally, we show that Gemin3 is capable of self-binding and Gem3 ${ }^{\Delta N}$, a helicase domain deletion mutant, enhances the association when bound to wild-type Gemin3, an observation that offers an explanation for its dominant-negative mechanism of action. Collectively, our data reinforce the link between SMA and ALS in addition to giving impetus to further studies on the shared mechanisms in a genetically tractable model organism.

\section{Results}

Overexpression of human TDP-43 in a Gem3 mutant background induces adult lethality. Similar to $\mathrm{SMN}^{45,62,63}$, loss of Gemin3 impacts adult viability and induces motor dysfunction ${ }^{64-66}$. In addition to Gemin3, a select number of SMN complex components, including Gemin2, Gemin4, Gemin5, Gemin8 and Unrip, are required for neuromuscular function and survival in Drosophila ${ }^{61,64,67}$. It is therefore highly plausible that SMA is triggered by any perturbation in the stoichiometry of the SMN complex. We have recently isolated Gem3 $3^{B A R T}$, a hypomorphic version of the Gem $3^{\Delta N}$ mutant, which lacks the N-terminal helicase core. Subsequently, we reported that alterations in the levels of SMN complex components precipitate the viability and motor phenotypes of Gem $3^{B A R T}$ adult flies ${ }^{68}$. A similar outcome was observed on disruption of snRNP biogenesis factors $\mathrm{pICln}$ and $\mathrm{Tgs} 1^{61}$. We wished to investigate whether a functional interaction also extends to Gemin3 and ALS-linked TDP-43. Missense mutations in this protein have been identified in $5 \%$ of familial and $<1 \%$ of sporadic ALS cases ${ }^{10}$. TDP-43, an evolutionarily conserved protein, comprises of 2 RNA recognition motifs (RRMs), a nuclear localisation signal and a nuclear export sequence that mediate nuclear shuttling, as well as a C-terminal glycine-rich region where the majority of ALS-associated mutations occur ${ }^{11}$. Importantly, truncated TDP-43 is mislocalised from its predominantly nuclear location to ubiquitin-containing cytoplasmic inclusions in neurons of both sporadic and most familial forms of ALS ${ }^{10,69,70}$. Loss of TDP-43 nuclear function has been proposed as a primary mechanism linking TDP-43 proteinopathy to neuromuscular degeneration in ALS. In this context, we first asked whether decreased levels of TDP-43 can modify Gem $3^{\text {BART }}$ phenotypes. We note that neither haploinsufficiency $\left(T B P H^{\triangle 23}\right)$ nor RNAi-induced knockdown (TBPH-RNAi) of TBPH, the Drosophila $T D P-43$ homologue, had any effect on motor and viability phenotypes in flies with muscle-restricted Gem $3^{\text {BART }}$ expression (Fig. 1, Table 1 and data not shown).

Recently, considerable attention has been given to the toxic effect of cytoplasmic TDP-43 protein aggregates ${ }^{71}$. To this end, we next queried whether gain rather than loss of TDP-43 function is a modifying factor. Expression of wild-type human TDP-43 (hTDP-43 ${ }^{\mathrm{WT}}$ ) in muscle leads to adult flies that have climbing defects and are entirely flightless when compared to controls (Fig. 1). Flies also have a shortened life-span, therefore surviving less than a 
A

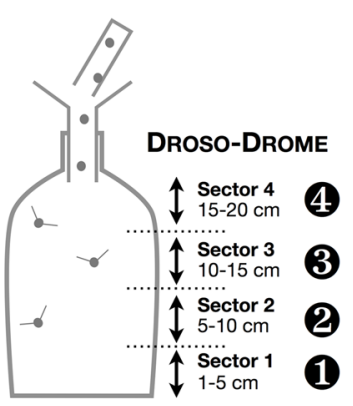

\begin{tabular}{|c|c|}
\hline Genotype & Line \\
\hline$\delta$ Mef2-GAL4 > +/TBPH ${ }^{\Delta 23}$ & $=$ \\
\hline$\delta$ Mef2-GAL4 > +/TBPH $H^{\triangle 23}+$ Gem3 $3^{B A R T}$ & $\infty$ \\
\hline$\delta$ Mef2-GAL4 > TBPH-RNAi [Trinity] & $\Rightarrow$ \\
\hline$\delta$ Mef2-GAL4 > TBPH-RNAi [Trinity] + Gem3 $3^{\text {BART }}$ & - \\
\hline$\delta h T D P-43^{W T} /+$ & $=$ \\
\hline$\delta$ Mef2-GAL4 > hTDP-43"WT & - \\
\hline 위 Mef2-GAL4 > hTDP-43 $3^{W T}+G e m 3^{B A R T}$ & Adult Lethal \\
\hline
\end{tabular}

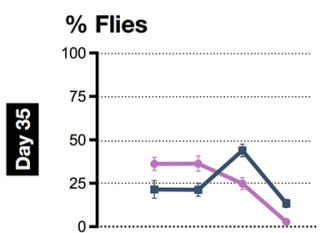

目
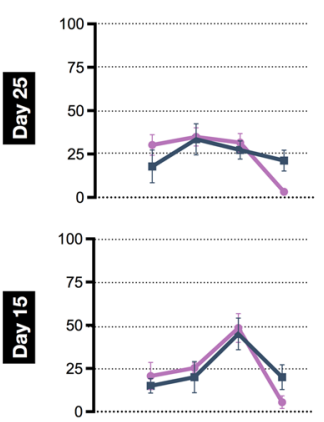

目

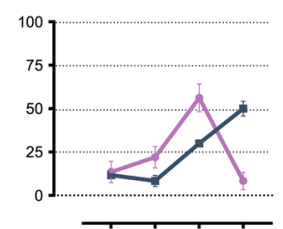

(1) 2 3 (4)

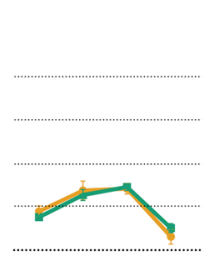

B Day 5
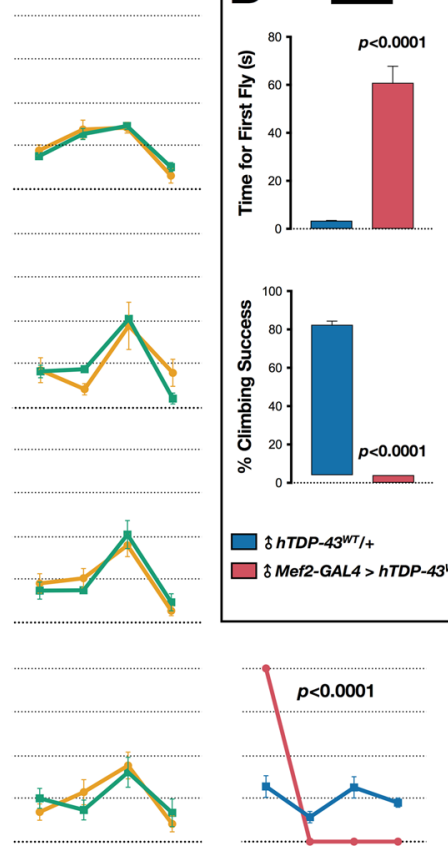

(1) 2 (3) 4

Droso-Drome Sector

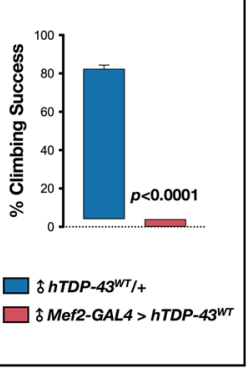

Figure 1. Gain-of-function identifies TDP-43 as a modifier of survival in Gem3 ${ }^{\text {BART }}$-expressing flies. (A) Left: Flight performance was assessed by the Droso-Drome apparatus, where determination of flight capacity is based on which sector flies land after they are introduced at the top. Right: Removal of one copy $\left(+/ T B P H^{\triangle 23}\right)$ or RNAi-mediated knockdown (TBPH-RNAi [Trinity]) of TBPH does not impair the motoric ability of flies with pan-muscular expression of Gem $3^{\text {BART }}$. Indeed, at all time points, no significant differences were observed between the two groups in the number of flies that had no flight ability (sector 1) compared to those that retained the ability to fly (sectors 2-4). In contrast, ectopic expression of wild-type human TDP-43 (hTDP$43^{\mathrm{WT}}$ ) in muscle impairs flight as early as day 5 post-eclosion. Flies were non-fliers, hence they all fell in sector 1. (B) Climbing success rate of flies with muscle-specific hTDP-43 overexpression was drastically reduced compared to control animals. Furthermore, assessment of the time taken for the first fly to reach a pre-set threshold determined that flies took significantly longer to attain this goal in contrast to the control genotype. In $(\mathbf{A}, \mathbf{B})$, data presented are the mean \pm S.E.M. of at least 4 independent experiments, and for each time point measured, $n \geq 60$ per genotype. Symbols indicate the sex of the genotype assessed: $\delta=$ males, $q=$ females, and 우 $=$ males + females. Significance as tested by two-way ANOVA, followed by Bonferroni's post hoc tests (A) and the unpaired $t$-test $(\mathbf{B})$ is indicated by the exact $p$-value.

week post-eclosion. Notably, in combination with Gem3 $3^{\text {BART }}$, hTDP-43 induced adult lethality with flies dying at the pupal stage. Furthermore, transgenes with higher expression levels and/or epitope-tagged versions of hTDP$43\left(h T D P-43^{W T} . G F P^{\# 10}\right.$, weak expression; $h T D P-43^{W T} . G F P^{\# 16}$, strong expression; ref. ${ }^{72}$ ) were found to induce death at the earlier third instar stage (L3) when combined with Gem $3^{B A R T}$ in contrast to death at pupal stage when they were expressed alone (Table 1). This trend, which was also observed with an independently-generated line (Flag.hTDP-43 ${ }^{W T}$, ref. ${ }^{73}$; Table 1), shows that phenotypic enhancement is dependent on dose or modifications that interfere with protein structure. Muscle-directed expression of hTDP- $43^{\text {CTF }}$, which mimics the major TDP-43 C-terminal fragment found in cytosolic aggregates of ALS patients, was found to induce lethality at the first instar larval stage most likely because the transgene is highly expressing ${ }^{72}$. This excluded its use for interaction analysis (Table 1). Importantly, overexpression of endogenous TBPH replicated the modifier effect of its human counterpart. Hence, whereas alone it induces death at the pupal stage, when combined with Gem $3^{\text {BART }}$ it enhanced survival defects with flies dying earlier than the L3 stage (Table 1). Overall, these findings are suggestive of a genetic interaction between Gemin3 and TBPH or its human homologue, TDP-43.

Knockdown or overexpression of caz/FUS enhances Gem3 mutant phenotypes. FUS is another major RBP that is mutated in both familial $(5 \%)$ and sporadic $(<1 \%)$ ALS cases ${ }^{10}$. FUS is a highly-conserved protein possessing an N-terminal domain rich in glutamine, glycine, serine and tyrosine residues (QGSY region), a glycine-rich region, an RRM, multiple arginine/glycine/glycine (RGG) repeats in an arginine- and glycine-rich region, and a zinc finger motif at the $\mathrm{C}$-terminus. Mutations cluster in the glycine-rich region and in the extreme C-terminus where the nuclear localisation signal is likely to reside ${ }^{11}$. Similar to TDP-43, mutant FUS is mislocalised to the cytoplasm where it forms ubiquitinated aggregates ${ }^{74}$. Considering that FUS and TDP-43 function 


\begin{tabular}{|c|c|c|c|c|}
\hline \multirow[b]{2}{*}{ ALS GENE } & \multirow[b]{2}{*}{ ALLELE } & \multirow[b]{2}{*}{ REF. } & \multicolumn{2}{|l|}{ VIABILITY } \\
\hline & & & Mef2-GAL4> & Mef2-GAL4 > + Gem3 $3^{\text {BART }}$ \\
\hline \multirow{12}{*}{ Hs: $T D P-43 ; \mathrm{Dm}: T B P H$} & LOF: $+/ T B P H^{\Delta 23}$ & 73 & Adult Viable & Adult Viable \\
\hline & LOF$^{1}: T B P H-R N A i[$ Trinity] & 73 & Adult Viable & Adult Viable \\
\hline & LOF$^{1}: T B P H-R N A i[$ Merton] & 73 & Adult Viable & Adult Viable \\
\hline & LOF$^{1}: T B P H-R N A i$ [Maudlin] & 126 & Death at $\mathrm{P}$ & Death at $P$ \\
\hline & $\mathrm{OE}^{2}: h T D P-43^{W T}$ & 72 & Adult Viable & Death at $\mathrm{P}$ \\
\hline & $\mathrm{OE}^{1}: h T D P-43^{W T} \cdot G F P^{\sharp 10}$ & 72 & Death at $\mathrm{P}$ & Death at L3 \\
\hline & $\mathrm{OE}^{1}: h T D P-43^{W T} \cdot G F P^{\# 16}$ & 72 & Death at $\mathrm{P}$ & Death at L3 \\
\hline & $\mathrm{OE}^{1}: h T D P-43^{C T F} \cdot G F P^{\sharp 14}$ & 72 & Death prior to L3 & N/A \\
\hline & $\mathrm{OE}^{1}$ : Flag.hTDP-43 ${ }^{W T}$ & 73 & Death at $\mathrm{P}$ & Death at L3 \\
\hline & $\mathrm{OE}^{1}$ : Flag.TBPH ${ }^{W T}$ & 73 & Death prior to L3 & N/A \\
\hline & $\mathrm{OE}^{2}: T B P H^{W T}$ & 75 & Death prior to L3 & N/A \\
\hline & $\mathrm{OE}^{2}:$ Venus-TBPH ${ }^{W T}$ & 75 & Death at $P$ & Death prior to L3 \\
\hline \multirow{11}{*}{ Hs: FUS; Dm: Caz } & LOF: $c a z^{1} /+^{*}$ & 75 & Adult Viable & Adult Viable \\
\hline & LOF $^{2}: c a z-R N A i[$ Kellogg] & 121,127 & Adult Viable & Adult Viable \\
\hline & LOF$^{2}:$ caz-RNAi [Oriel] & N/A & Adult Viable & Adult Viable \\
\hline & $\mathrm{OE}^{2}$ : Flag.caz ${ }^{W T}$ & 75 & Adult Viable & Death at $P$ \\
\hline & $\mathrm{OE}^{2}$ : Flag.caz ${ }^{\text {P398L }}$ & 75 & Death at $\mathrm{P}$ & Death at $P$ \\
\hline & $\mathrm{OE}^{2}:$ Flag.hFUS ${ }^{W T}$ & 75 & Adult Viable & Death prior to L3 \\
\hline & $\mathrm{OE}^{2}: H A . h F U S^{W T}$ & 128 & Death at $\mathrm{P}$ & Death at $\mathrm{P}$ \\
\hline & $\mathrm{OE}^{1}: h F U S^{P 525 L}-R F P . H A$ & 129 & Death prior to L3 & N/A \\
\hline & $\mathrm{OE}^{1}: h F U S^{W T}$-RFP.HA & 129 & Death prior to L3 & N/A \\
\hline & $\mathrm{OE}^{1}:$ hFUS ${ }^{R 524 S}-$ RFP.HA & 129 & Death at $\mathrm{P}$ & Death at $\mathrm{P}$ \\
\hline & $\mathrm{OE}^{2}$ : Flag.hFUS ${ }^{P 525 L}$ & 75 & Death at $P$ & Death at L3 \\
\hline \multirow{6}{*}{ Hs: $C 9$ orf72 } & $\mathrm{OE}^{2}: G_{4} C_{2}-3$ & 123 & Adult Viable & Adult Viable \\
\hline & $\mathrm{OE}^{2}: G_{4} C_{2}-36$ & 123 & Adult Viable & Adult Viable \\
\hline & $\mathrm{OE}^{2}: G R-36$ & 123 & Adult Viable & Adult Viable \\
\hline & $\mathrm{OE}^{2}: G R-100$ & 123 & Death prior to L3 & N/A \\
\hline & $\mathrm{OE}^{2}: P R-36$ & 123 & Adult Viable & Adult Viable \\
\hline & $\mathrm{OE}^{2}: P R-100$ & 123 & Adult Viable & Adult Viable \\
\hline \multirow{8}{*}{ Hs: SOD1; Dm: Sod1 } & LOF: $\operatorname{Sod} 1^{n 1} /+^{*}$ & 80 & Adult Viable & Adult Viable \\
\hline & LOF$^{2}$ : Sod1-RNAi [Pembroke] & 122 & Adult Viable & Adult Viable \\
\hline & LOF$^{1}$ : Sod1-RNAi [Hertford] & 122 & Adult Viable & Adult Viable \\
\hline & $\mathrm{OE}^{1}: h S O D 1^{W T}$ & 130 & Adult Viable & Adult Viable \\
\hline & $\mathrm{OE}^{2}: h S O D 1^{W T} \cdot H A$ & 131 & Adult Viable & Adult Viable \\
\hline & $\mathrm{OE}^{1}: h S O D 1^{G 85 R}$ & 130 & Adult Viable & Adult Viable \\
\hline & $\mathrm{OE}^{1}: h S O D 1^{A 4 V}$ & 130 & Adult Viable & Adult Viable \\
\hline & $\mathrm{OE}^{1}:$ Sod1 & 130 & Adult Viable & Adult Viable \\
\hline
\end{tabular}

Table 1. Alleles of ALS-linked genes investigated in this study and their effect on viability when expressed either alone or in combination with Gem $3^{B A R T}$ in muscle tissue. ${ }^{1}$ Transgenesis: random insertion; ${ }^{2}$ Transgenesis: $\Phi$-C31 site-specific insertion; *Heterozygote; Hs, Homo sapiens (human); Dm, Drosophila melanogaster (fruit fly); N/A = Not Applicable; L3, third instar larval stage; P, pupal stage, LOF, loss of function; OE, overexpression.

in a common pathway with FUS acting downstream of TDP- $43^{75,76}$, we hypothesised that Gemin 3 is likely to have a functional relationship not only with TBPH/TDP-43 but also with FUS. To this end, we first tested whether haploinsufficiency of cabeza (caz), the Drosophila homologue of FUS, can induce motor deficits when placed in a Gem $3^{B A R T}$ genetic background. Interestingly, we find a subtle yet statistically significant difference in motoric abilities at late adulthood (day 35 post-eclosion) in $c a z$ mutant heterozygous flies $\left(\mathrm{caz}^{1} /+\right)$ that had muscle-restricted Gem3 $3^{B A R T}$ expression compared to those that did not (Fig. 2). Subsequently, we asked whether phenotypic enhancement is dose-dependent. Hence, we induced muscle-specific RNAi-mediated knockdown of caz in wild-type versus Gem $3^{\text {BART }}$ flies. We observed that a moderately-expressing RNAi transgene targeting the C-terminus (caz-RNAi [Kellogg], Supplementary Fig. S1) induced flight defects as early as day 15 post-eclosion with flies then exhibiting an age-dependent progressive worsening in phenotype (Fig. 2). A stronger RNAi transgene targeting the same region but based on short hairpin microRNA (shRNA) technology (caz-RNAi [Oriel], Supplementary Fig. S1) was capable of inducing motor defects at an earlier stage during adulthood (Fig. 2), further confirming that modification is dependent on Caz protein levels with a severe reduction inducing the highest impact.

Similar to TDP-43, in addition to loss of nuclear function, a toxic gain of function due to the formation of cytoplasmic aggregates has been implicated as a predominant mechanism underpinning FUS-associated 

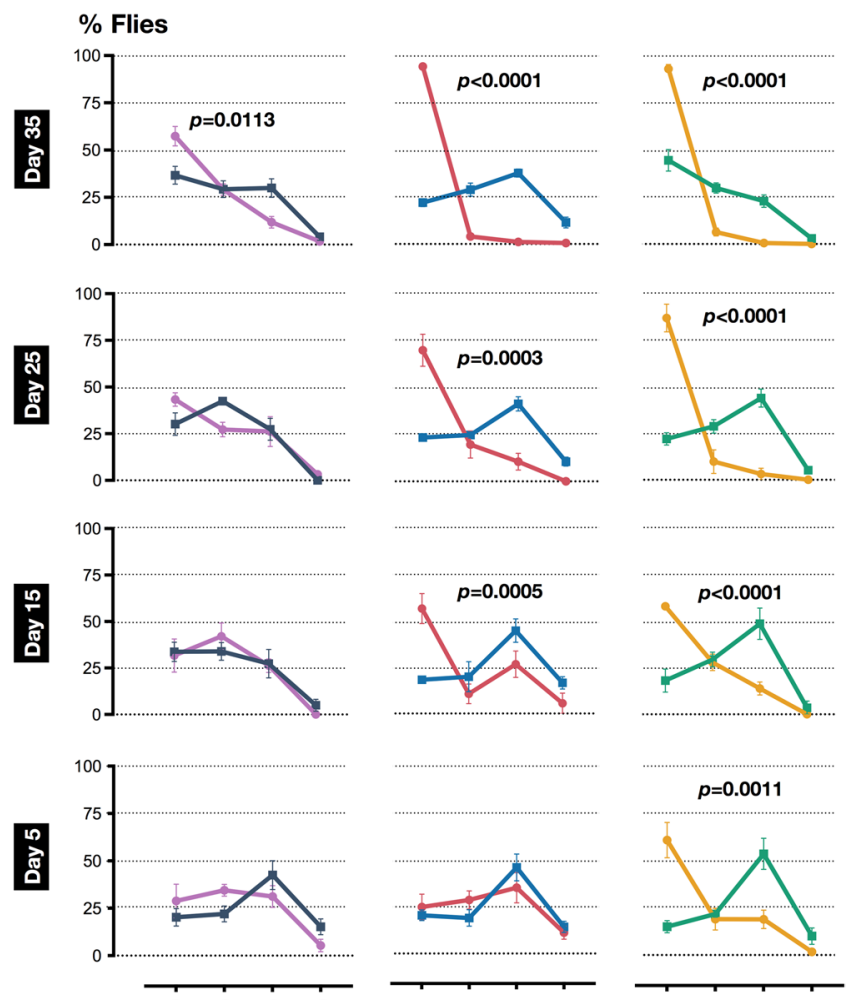

\section{(1) 238

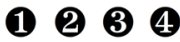 \\ (1) 2384 \\ Droso-Drome Sector}

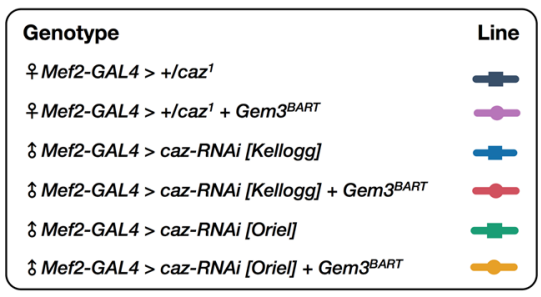

Figure 2. Loss of caz function impacts neuromuscular ability in Gem3 mutant flies. Left panel: In a heterozygous caz deficient background, brought about by the $c a z^{1}$ null mutant, Gem $3^{B A R T}$ flies develop flight defects at a late stage during adulthood. Hence, motor deficits become obvious only on day 35 post-eclosion. Middle panel: A greater reduction in Caz levels induced by a moderately-expressing RNAi transgene (caz$R N A i$ [Kellogg]) induced flight defects at an earlier stage (day 15 post-eclosion) and an increase in severity was observed with age. Right panel: A more pronounced age-dependent progressive decline in flight capacity can be brought about by knockdown mediated by a stronger RNAi transgene (caz-RNAi [Oriel]). Data presented are the mean \pm S.E.M. of at least 4 independent experiments, and, for each time point measured, $n \geq 60$ per genotype. Symbols indicate the sex of the genotype assessed: $\delta=$ males, and $q=$ females. Significance as tested by two-way ANOVA, followed by Bonferroni's post hoc tests is indicated by the exact $p$-value.

pathophysiology ${ }^{74,77}$. In this context, we questioned whether upregulation of $c a z$ or overexpression of human FUS (hFUS ${ }^{\mathrm{WT}}$ ) can also act as enhancers when placed in a Gem 3 mutant background. In this regard, in a wild-type background, muscle-restricted increase in Caz protein levels was sufficient to induce both climbing and flight defects as early as day 5 post-eclosion (Fig. 3). However, in Gem $3^{\text {BART }}$ flies, $c a z^{W T}$ upregulation induced lethality before eclosion with the majority of flies dying during the pupal stage (Table 1). Similarly, ectopic expression of hFUS $^{W T}$ in muscles was enough to cause motor deficits in young adult flies when applied to a wild-type background (Fig. 3). In combination with Gem $3^{\text {BART }}$, hFUS ${ }^{\mathrm{WT}}$ overexpression remarkably induced death during early development (Table 1). A trend towards reduced survival was also observed in Gem $3^{\text {BART }}$ flies upon expression of hFUS with a pathogenic mutation in the C-terminus $\left(h F U S^{P 525 L}\right)$, though not when expressing $c a z^{P 398 L}$, its equivalent in Drosophila (Table 1). Collectively, these findings show that either loss or gain of Caz/FUS function aggravate the motor and viability phenotypes of Gem3 mutant flies, which is highly suggestive of a genetic association between Gemin 3 and caz/FUS.

Expression of C9orf72 repeat expansions has no effect on Gem3 mutant flies. We next sought to broaden our investigation by determining whether Gem3 mutant phenotypes are also induced by disruption 
A
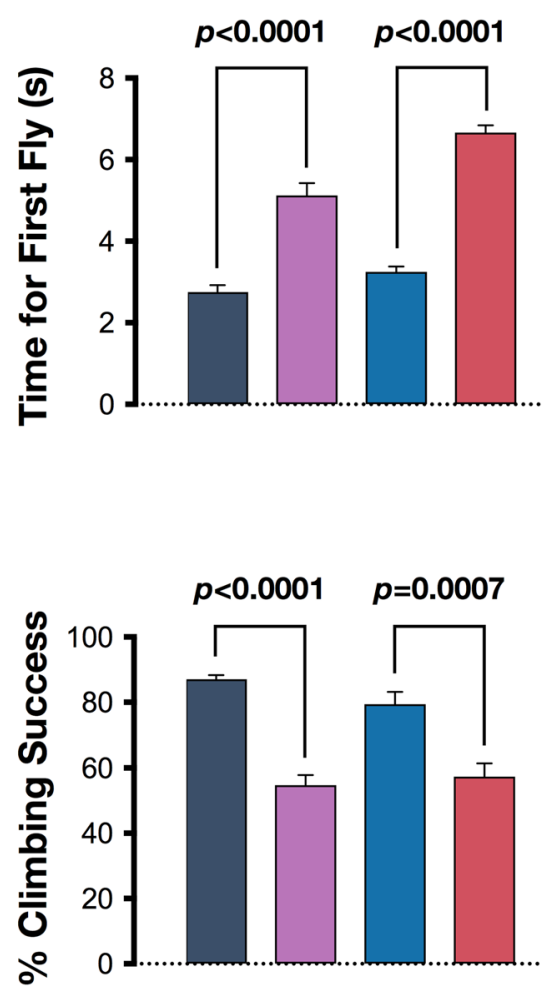

B

\section{$\%$ Flies}

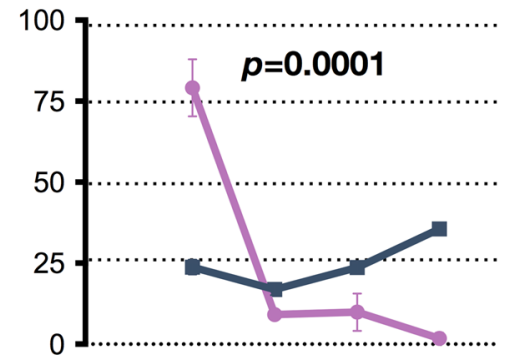

Sector: 1

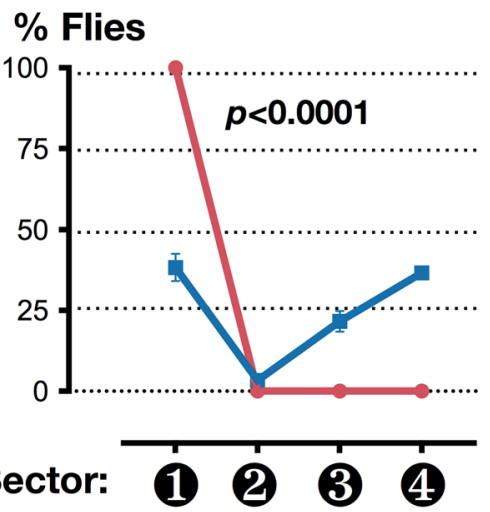

\begin{tabular}{|c|c|}
\hline Genotype & Bar Line \\
\hline 今 Flag.caz ${ }^{W T /+}$ & \\
\hline 今 Mef2-GAL4 > Flag.caz ${ }^{W T}$ & \\
\hline 웅 Mef2-GAL4 > Flag.caz ${ }^{W T}+$ Gem3 $3^{B A R T}$ & Adult Lethal \\
\hline 今 Flag.FUSWT/+ & \\
\hline 今 Mef2-GAL4 > Flag.FUS ${ }^{W T}$ & 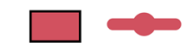 \\
\hline 웃 Mef2-GAL4 > Flag.FUSWT + Gem3 $3^{B A R T}$ & Adult \\
\hline
\end{tabular}

Figure 3. Gain of caz/FUS function in muscle impairs motor performance. (A) Overexpression of either caz or human FUS ( $h F U S$ ) in muscle leads to adult flies with reduced mobility. Hence, on assessment, the first fly took significantly longer to reach a pre-set threshold. Furthermore, at a population level, climbing success was profoundly reduced. (B) Flies were in their majority non-fliers. Thus, when tested, a significant percentage dropped to the base (sector 1) of the Droso-Drome. Importantly, in combination with Gem $3^{B A R T}$, muscle-specific overexpression of either $c a z$ or $h F U S$ induced lethality prior to eclosion. Data presented are the mean \pm S.E.M. of at least 4 independent experiments, and for each time point measured, $n \geq 60$ per genotype.

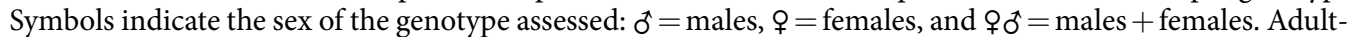
viable flies were assessed at day 5 post-eclosion. Significance as tested by the unpaired $t$-test (A) and two-way ANOVA, followed by Bonferroni's post hoc tests $(\mathbf{B})$ is indicated by the exact $p$-value.

of other major ALS-linked genes. C9orf72 is the most frequently mutated gene in ALS. Enormous expansions of an intronic hexanucleotide repeat (GGGGCC, $\mathrm{G}_{4} \mathrm{C}_{2}$ ) cause a large portion of familial $(25 \%)$ and sporadic $(10 \%)$ $\mathrm{ALS}^{10}$. Transcripts containing repeats form intranuclear RNA foci that sequester nuclear proteins. In the cytoplasm, expanded RNA also undergoes repeat-associated non-AUG (RAN) translation to produce toxic dipeptide repeat (DPR) proteins ${ }^{9}$. Transgenic expression of a non-pathogenic repeat length $\left(\mathrm{G}_{4} \mathrm{C}_{2}-3\right)$ in muscle tissue had no effect on neuromuscular function in either wild-type or Gem $3^{B A R T}$ flies. Thus, at all time points assessed, flies were relatively healthy and no major differences were apparent between the two groups at any stage during their adult life (Fig. S2). Although muscle-restricted expression of 36 repeats $\left(\mathrm{G}_{4} \mathrm{C}_{2}-36\right)$, previously shown to be neurotoxic $^{78}$, caused flight defects in early adulthood, the phenotype in $G e m 3^{B A R T}$ flies was surprisingly identical to that of control flies with a wild-type background (Fig. S2). It is noteworthy that a similar outcome was observed in either background when assessing animals expressing two arginine-containing DPR proteins, glycine-arginine 
(GR-36) or proline-arginine (PR-100) (Fig. S2). In addition to confirming that repeats are damaging through the production of DPR proteins ${ }^{78}$, this result underscores that neither expanded repeats nor poly-GR/poly-PR proteins enhance Gem3 loss-of-function.

Loss rather than gain of Sod1 function enhances motor deficits in Gem3 mutant flies. The first ALS gene to be identified was SOD1, which encodes for the $\mathrm{Cu}-\mathrm{Zn}$ superoxide dismutase, an abundant ubiquitously-expressed cytoplasmic enzyme. SOD1 is the second most commonly mutated gene in ALS, contributing to $20 \%$ and $2 \%$ of familial and sporadic cases, respectively ${ }^{10}$. SOD 1 performs an important antioxidant function by catalysing the conversion of highly reactive superoxide to hydrogen peroxide or oxygen. Nevertheless, neuromuscular degeneration is thought to be driven by one or more acquired toxicities of the mutant protein rather than loss of dismutase activity. Indeed, similar to TDP-43 and FUS, most ALS-causing SOD1 mutants form ubiquitinated cytoplasmic aggregates that are toxic to various cellular processes ${ }^{79}$. Against this backdrop, we investigated whether gain of Sod 1 function is also capable of triggering motor dysfunction in Gem 3 mutant flies. We found that neither overexpression of Drosophila Sod1 nor ectopic expression of wild-type human SOD1 $\left(\mathrm{hSOD} 1^{\mathrm{WT}}\right.$ ) had any negative effect in either a wild-type or a Gem3 mutant background (Fig. 4). Expression of pathogenic variants including hSOD $1^{\mathrm{A} 4 \mathrm{~V}}$ or hSOD $1^{\mathrm{G} 85 \mathrm{R}}$ gave a similar result, hence they were not damaging in either genetic background (Fig. 4). Interestingly, we were surprised to note that less than $50 \%$ reduction in enzymatic activity, brought about by heterozygosity for the missense allele Sod $1^{G 51 S}\left(+/ \operatorname{Sod} 1^{G 51 S}\right)^{80}$, induced a prominent decrease in neuromuscular function during late adulthood in Gem 3 mutant flies, suggesting that these organisms are susceptible to oxidative stress when they get old. We confirmed this result through the use of muscle-specific RNAi-mediated loss of Sod 1 function in wild-type versus Gem $3^{B A R T}$ flies. Hence, we show that an RNAi transgene targeting the C-terminus (Sod1-RNAi [Pembroke], Supplementary Fig. S1) similarly provoked flight defects in adult flies aged to day 35 post-eclosion (Fig. 5). Motor defects became apparent at an even earlier stage (day 25 post-eclosion) when we made use of a stronger RNAi transgene targeting the same region but having a longer hairpin sequence (Sod1-RNAi [Hertford], Fig. 5 and Supplementary Fig. S1). Overall, these findings demonstrate that Gem 3 mutant phenotypes are hastened by Sod 1 loss-of-function rather than by gain-of-function, hence allowing us to uncover a genetic interaction between Gemin3 and Sod1.

TDP-43 or FUS gain of function enhance muscle atrophy and suppresses neuromuscular junction overgrowth in Gem3 mutant flies. Above we showed that upregulation of $c a z$ or TBPH, in flies with Gem3 loss of function, induced adult lethality. Overexpression of the respective human homologue gave an analogous outcome. We next investigated whether the neuromuscular function of these animals is perturbed prior to their death. Gem $3^{\text {BART }}$ animals devoid of any genetic modifying factor(s) eclose normally and neuromuscular function is relatively unperturbed in adult flies. Surprisingly, when analysing larval crawling, we observed a slight yet significant decline in mobility in Gem3 $3^{B A R T}$ larvae compared to their wild-type counterparts (Fig. 6A). Notably, this difference can be explained by a substantial difference in muscle surface area between Gem 3 mutant and control larvae. Thus, the former had a pronounced reduction in muscle size (Fig. 6B). We asked whether these phenotypes are amenable to modification by genetic factors. To this end, we introduced Gaulos RNAi in Gem3 mutants. Gaulos (Glos) was recently identified as the Drosophila orthologue of Gemin $4^{67,81}$, a putative co-factor of Gemin $3^{82}$. Remarkably, muscle-driven Glos reduction caused a further decline in both the locomotor ability (Fig. 6A) and muscle size of Gem $3^{B A R T}$ larvae (Fig. 6B), hence confirming that the phenotypes can be genetically enhanced. Importantly, we demonstrate that compared to the baseline provided by Gem $3^{B A R T}$ larvae, ectopic expression of either hTDP-43 or hFUS was responsible for an additional degree of sluggishness in larvae as demonstrated by their less frequent movements (Fig. 6A). Interestingly, the difference was reflected in muscle size, hence muscle atrophy was greatly enhanced upon TDP-43 or FUS gain-of-function (Fig. 6B). Upregulation of TBPH causes Gem $3^{B A R T}$ flies to die before the third instar larval stage (Table 1), hence precluding assessment of flies. Upregulation of $c a z$ in Gem $3^{B A R T}$ flies induces larval mobility defects but has no effect on muscle size (data not shown). However, overexpression of the pathogenic variant $c a z^{P 398 L}$ in $G e m 3^{B A R T}$ flies was found to mirror the neuromuscular phenotypes observed on TDP-43/FUS gain of function in the same genetic background (Fig. 6A,B).

Consequent to muscle atrophy and the subsequent decline in muscle power, all genotypes assessed failed to contract adequately during pupariation. Hence, in a Gem $3^{B A R T}$ genetic background, Glos knockdown (serving as a positive control) or overexpression of $h T D P-43 / h F U S / c a z^{P 398 L}$, all induced a puparial axial ratio that was significantly higher than the baseline observed in Gem3 mutant flies devoid of any genetic manipulation (Fig. 6C). Finally, we assessed the morphology of the neuromuscular junction (NMJ). Muscle-directed expression of Gem $3^{\mathrm{BART}}$ causes an appreciative expansion of the NMJ (Fig. 7A), hence, parameters including area (Fig. 7B), number of branches (Fig. 7C) and bouton numbers (Fig. 7D) were all significantly elevated compared to the wild-type control. This phenotype was similar to that previously reported for flies with homozygous Gem3 loss-of-function in all tissues ${ }^{65}$. NMJ overgrowth was also observed on Glos knockdown and upon expression of $c a z^{P 398 L}$, both of them directed to muscle (Fig. 7). No deviations from the wild-type NMJ parameters were however seen on expression of either hTDP-43 or hFUS in muscle (Fig. 7). Interestingly, in a Gem $3^{\text {BART }}$ background, expression of $h T D P-43, h F U S$ and, to a slightly lower degree, caz $z^{P 398 L}$ (but not Glos RNAi) suppresses the NMJ overgrowth phenotype associated with Gem3 loss-of-function, with key NMJ morphology parameters reduced to the wild-type range (Fig. 7). In sum, these observations strengthen the evidence favouring the possibility that Gemin 3 acts together with TBPH/TDP-43 and caz/FUS within a genetic pathway that influences viability and neuromuscular function. 

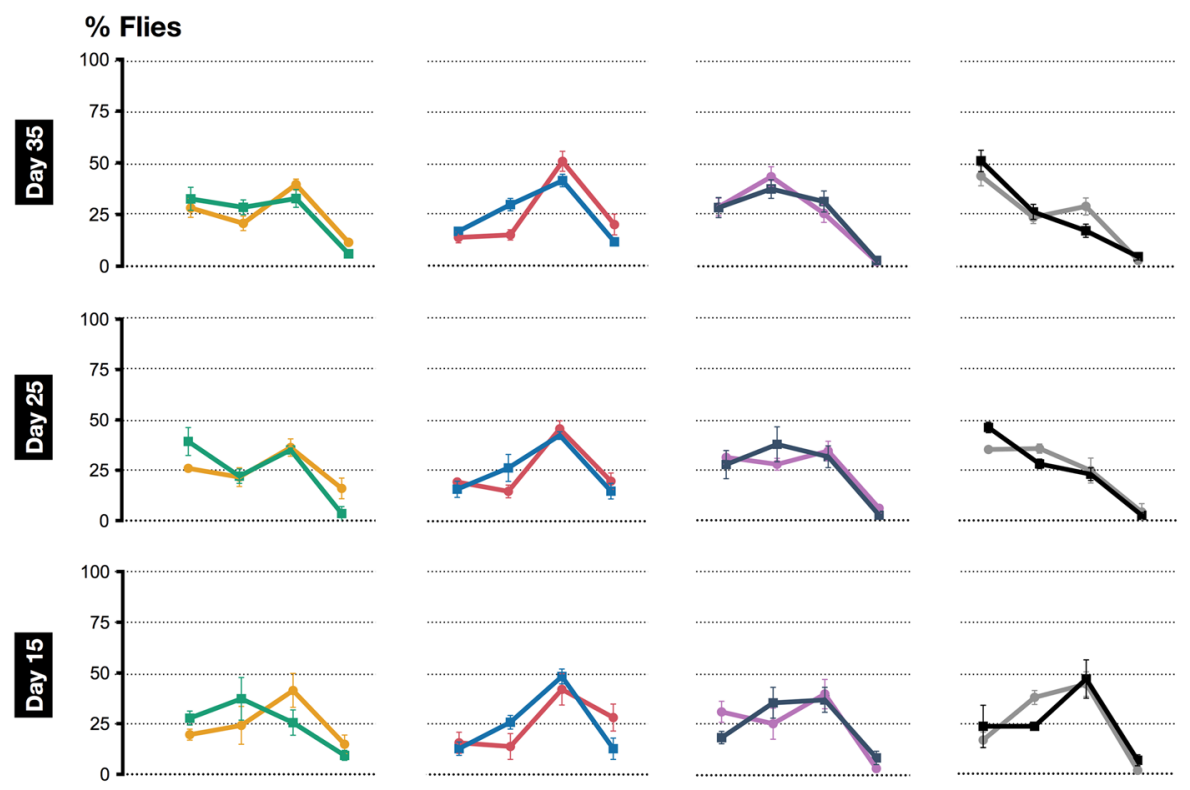

ำ
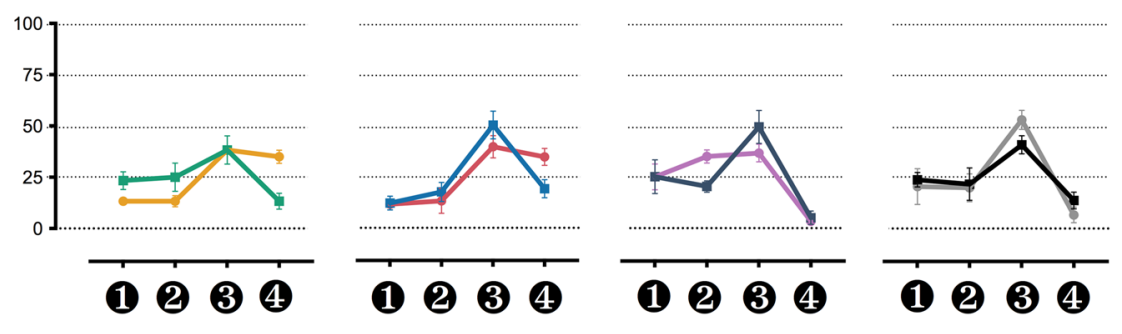

Droso-Drome Sector

\begin{tabular}{|c|c|}
\hline Genotype & Line \\
\hline$\hat{\delta}$ Mef2-GAL4 > Sod 1 WT & $\therefore$ \\
\hline$\delta$ Mef2-GAL4 > Sod1 $1 W T+G e m 3^{B A R T}$ & 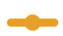 \\
\hline o Mef2-GAL4 > hSOD1 ${ }^{W T} T_{-H A}$ & \\
\hline$\delta$ Mef2-GAL4 > hSOD1 ${ }^{W T}-H A+G e m 3^{B A R T}$ & 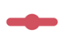 \\
\hline$\hat{\delta}$ Mef2-GAL4 > hSOD1A4V & $\therefore$ \\
\hline$\delta$ Mef2-GAL4 > hSOD1 $1^{A 4 V}+G e m 3^{B A R T}$ & $\propto$ \\
\hline o Mef2-GAL4 > hSOD1 $1^{\mathrm{G} 85 R} \times 4$ & $\rightarrow$ \\
\hline I Mef2-GAL4 > hSOD1 ${ }^{G 85 R} X 4+G e m 3^{B A R T}$ & \\
\hline
\end{tabular}

Figure 4. Sod 1 gain-of-function has no effect on motor behaviour in Gem3 mutant flies. Overexpression of either Sod1 or human SOD1 ( $h S O D 1$ ) is not deleterious to animals with a marginal loss of Gem3 function (left panel). Overexpression of pathogenic hSOD1 A4V or G85R variants is equally not a damaging factor to Gem3 mutant flies (right panel). Organisms did not show differences in motor function at all time points assessed.

Data presented are the mean \pm S.E.M. of at least 4 independent experiments, and, for each time point measured, $n \geq 60$ males ( $\left.0^{*}\right)$ per genotype.

Self-association of full-length Gemin3 explains loss of function induced by the Gem3 ${ }^{\text {BART }}$ mutant. In conclusion, we wished to gain insights into the mode of action of the Gem $3^{B A R T}$ allele, a hypomorphic or weaker version of the Gem $3^{\Delta N}$ mutant. The latter differs from wild-type Gemin 3 in that it lacks the $\mathrm{N}$-terminus which hosts the helicase domains ${ }^{68}$. Previously, we have shown that expression of Gem $3^{\Delta N}$ mimics Gem3 knockdown and, together, the genetic alterations cause lethality. This allowed us to conclude that expression of Gem $3^{\Delta N}$ in a wild-type background induces a loss of Gemin 3 function ${ }^{64}$. We hypothesised that $G e m 3^{\Delta N}$ interacts with its wild-type full-length counterpart and in so doing, it interferes with its function. This model assumes that Gemin 3 is capable of self-association for which the evidence is presently lacking. Through a yeast two-hybrid assay, we confirm this model, hence, we show that Drosophila Gemin3 is able to strongly interact with itself (Fig. 8A). Although this property is lost with $G e m 3^{\Delta N}$, remarkably, we demonstrate that $G e m 3^{\Delta N}$ is capable of binding to its full-length counterpart (Fig. 8A).

We next questioned whether the Gem $3^{\Delta N_{-}}$Gem 3 interaction is stronger than the one between wild-type Gemin3 monomers, hence favouring the capture of endogenous Gemin3 into non-functional oligomers. To this end, we re-performed the yeast two-hybrid assay in the presence of 3-amino-1,2,4-triazole (3-AT). 3-AT 

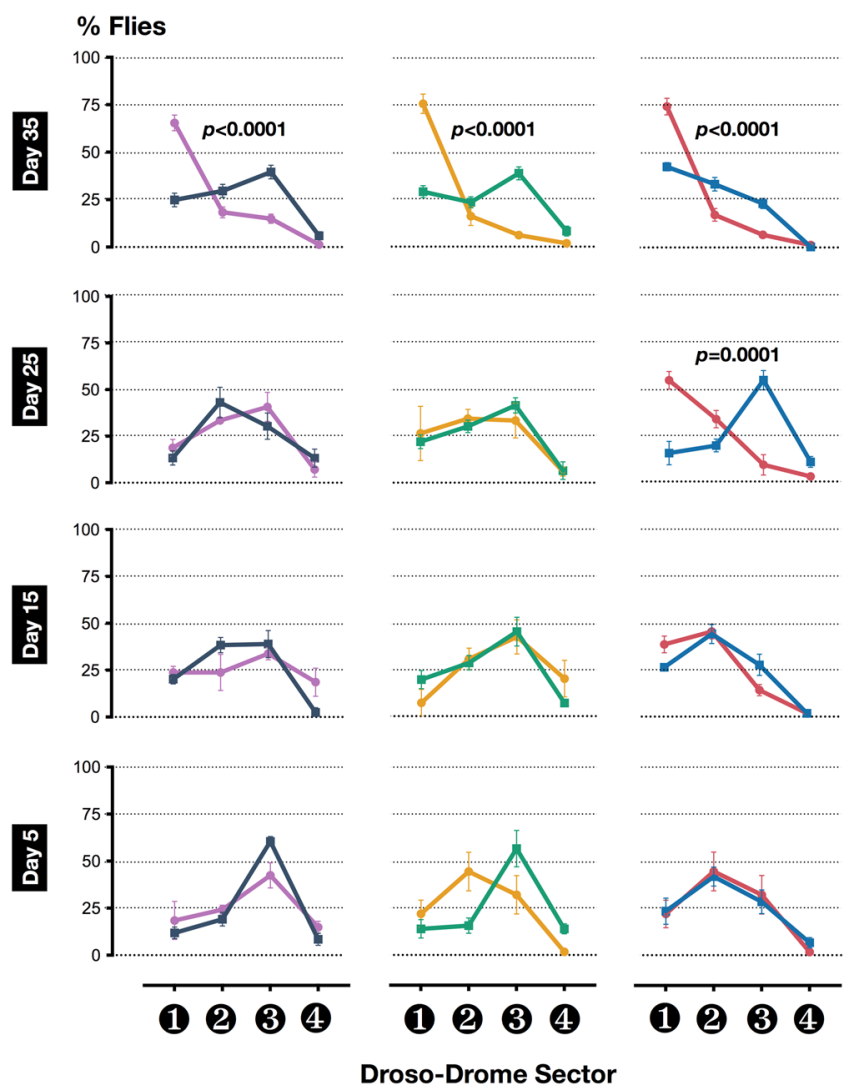

(1) 234

Droso-Drome Sector

\begin{tabular}{|c|c|}
\hline Genotype & Line \\
\hline$\hat{\delta}$ Mef2-GAL4 > +/Sod $11^{\mathrm{G}} 51 \mathrm{~S}$ & 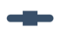 \\
\hline$\hat{o}$ Mef2-GAL4 > +/Sod1 $1^{\mathrm{G} 51 \mathrm{~S}}+\mathrm{Gem} 3^{\mathrm{BART}}$ & 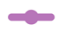 \\
\hline ó Mef2-GAL4 > Sod1-RNAi [Pembroke] & $\rightarrow$ \\
\hline I Mef2-GAL4 > Sod1-RNAi [Pembroke] + Gem3 $3^{\text {BART }}$ & $\rightarrow$ \\
\hline I Mef2-GAL4 > Sod1-RNAi [Hertford] & $\rightarrow$ \\
\hline f Mef2-GAL4 > Sod1-RNAi [Hertford] + Gem3 $3^{B A R T}$ & $\infty$ \\
\hline
\end{tabular}

Figure 5. Loss of Sod 1 function impairs motor behaviour in Gem 3 mutant flies. Placed in a Gem $3^{B A R T}$ genetic background, heterozygotes for an enzymatic null Sod 1 allele $\left(+/ S o d 1^{G 51 S}\right)$ only induce deficits in old age (left panel). A similar outcome is achieved in Gem3 $3^{\text {BART }}$ flies with muscle-selective RNAi-mediated knockdown of Sod1 (middle panel). The use of a stronger RNAi transgene causes defects at an earlier stage of adult life (right panel). Data presented are the mean \pm S.E.M. of at least 4 independent experiments, and, for each time point measured, $n \geq 60$ males ( $₫$ ) per genotype. Significance as tested by two-way ANOVA, followed by Bonferroni's post hoc tests, is indicated by the exact $p$-value.

competitively inhibits imidazole glycerol-phosphate dehydratase, a histidine (His) biosynthetic enzyme, thus limiting His synthesis ${ }^{83,84}$. We demonstrate that yeast containing both the Gem3 bait and the Gem3 ${ }^{\Delta N}$ prey were capable of growing on -Leu-Trp-His selective plates at $20 \mathrm{mM} 3$-AT (Fig. 8B), thus indicating that the two-hybrid Gem3-Gem $3^{\Delta N}$ interaction is strong enough to overcome the growth inhibitory effect of 3-AT in the medium. In summation, these findings are first supportive of Gemin 3 self-binding. Second, they allow us to postulate that the dominant-negative nature of $G e m 3^{\Delta N}$ arises from its most-favourable binding to endogenous Gemin3. This is predicted to titre wild-type Gemin3 into non-functional dimers or oligomers.

\section{Discussion}

In this study, we sought to determine whether a functional interaction exists between Gemin3, a core SMN complex component, and major ALS-associated proteins. Focusing on motor behaviour, muscle mass, NMJ structure and survival, which are all profoundly affected in motor neuron disease, we show that disruption of either TBPH/ TDP-43 or Caz/FUS enhance muscle defects but are able to suppress NMJ morphology deficits, both induced by Gemin 3 loss-of-function. We also found that depletion of Sod 1 has an enhancing effect on neuromuscular function in old age. In addition to highlighting shared pathways most likely involving aspects of ribostasis and oxidative stress, our findings reinforce the link between SMA and ALS. Importantly, they extend our knowledge on the function of Gemin3, showing for the first time that it self-interacts, which is a property that makes it prone to loss of function. 
A
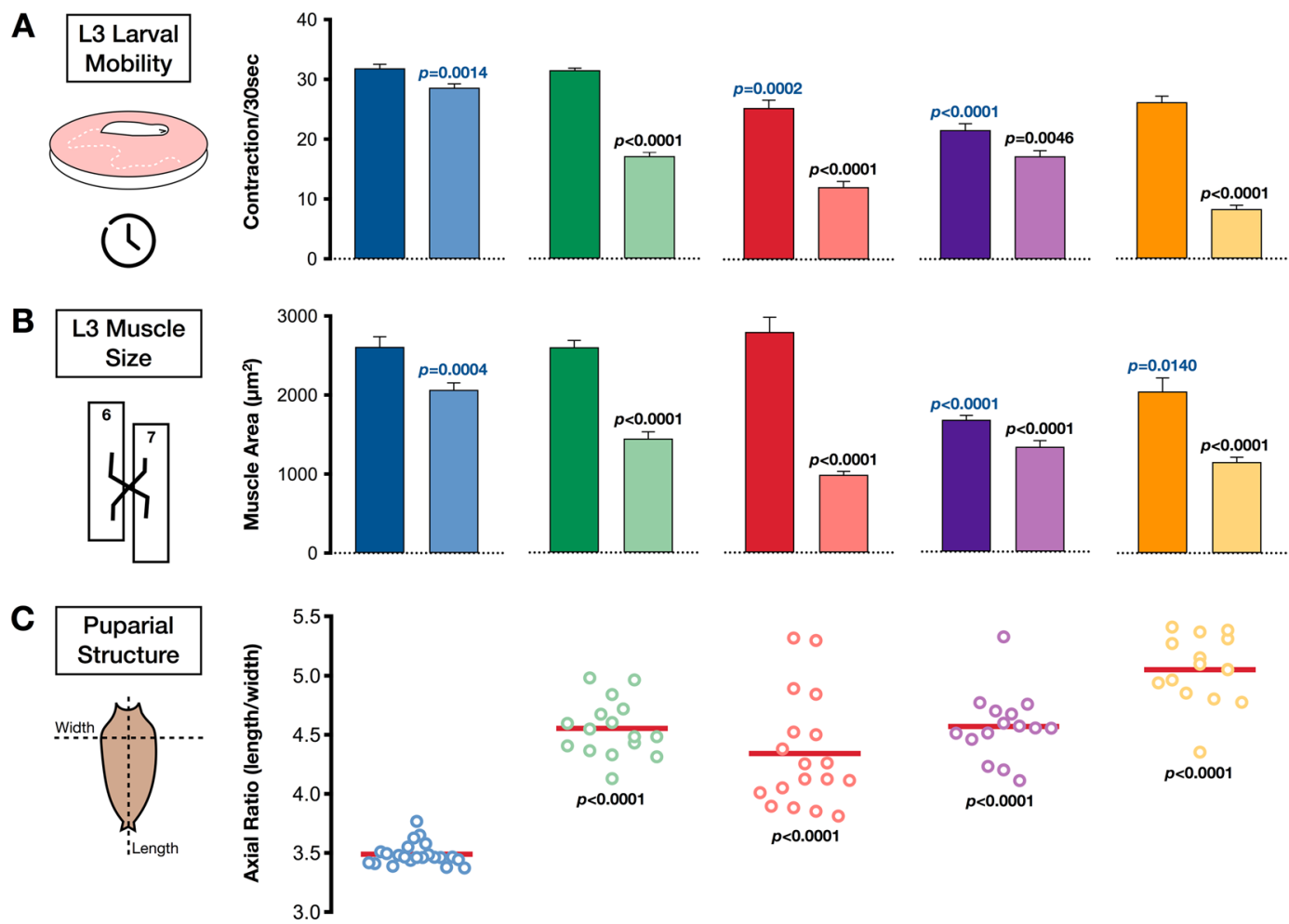

\begin{tabular}{|c|c|c|}
\hline Genotype & Bar & Symbol \\
\hline Wild-type & & \\
\hline Mef2-GAL4 > Gem3 $3^{B A R T}$ & & ○ \\
\hline Mef2-GAL4 > Glos RNAi & & \\
\hline Mef2-GAL4 > Gem3 $3^{B A R T}+$ Glos RNAi & $\square$ & ० \\
\hline Mef2-GAL4 > hTDP-43WT & ᄃ & \\
\hline$M e f 2-G A L 4>G e m 3^{B A R T}+h T D P-43^{W T}$ & $\square$ & ० \\
\hline Mef2-GAL4 > hFUS ${ }^{W T}$ & & \\
\hline Mef2-GAL4 > Gem3 $3^{B A R T}+h F U S^{W T}$ & $\square$ & ० \\
\hline Mef2-GAL4 > caz ${ }^{P 398 L}$ & L & \\
\hline Mef2-GAL4 > Gem3 $3^{B A R T}+\mathrm{caz}^{\text {P39gL }}$ & $\square$ & $\circ$ \\
\hline
\end{tabular}

Figure 6. Overexpression of hTDP-43 or hFUS/caz ${ }^{P 398 L}$ in Gem 3 mutant flies causes early mobility defects, reduced muscle size and aberrant puparial structures. (A) Third instar larvae with muscle-specific Gem3 $3^{\text {BART }}$ expression have a significant reduction in velocity compared to wild-type controls. This deficit was profoundly enhanced on overexpression of $h T D P-43^{W T}, h F U S^{W T}$ or $c a z^{P 398 L}$. A similar result was obtained on knockdown of Gaulos (Glos RNAi), which served as a positive control. In the absence of Gem3 $3^{\mathrm{BART}}$, only overexpression of $h T D P-43^{W T}$ or $h F U S^{W T}$ was found to induce a decrease in larval mobility compared to wild-type animals. (B) Compared to the wild-type control, Gem $3^{B A R T}$ larvae also show a significant reduction in muscle size that dramatically worsens on Glos knockdown or upon overexpression of $h T D P-43^{W T}, h F U S^{W T}$, or $c a z^{P 398 L}$, all directed to muscle. In a wild-type background, muscle size was also found reduced in larvae with Glos knockdown or those overexpressing FUS/caz ${ }^{P 398 L}$. (C) Sluggish larval behaviour leads to the formation of puparia that have a significantly higher axial ratio (defined as length/width) compared to the baseline offered by Gem $3^{B A R T}$ larvae. In $(\mathbf{A}-\mathbf{C})$ data presented are the mean \pm S.E.M. of at least 3 independent experiments, and $n \geq 15$ per genotype. Equal number of male and female larvae were used in each assay. Significance as tested by the unpaired $t$-test is indicated by the exact $p$-value, shown either in blue (comparison to wild-type larvae) or black (comparison to Gem $3^{B A R T}$ larvae).

Defective chaperoning of spliceosome assembly and/or missplicing have long been known to have a major role in the pathophysiology of motor neuron degeneration. A plethora of in vivo studies unequivocally show that snRNP assembly defects and the consequential missplicing events can induce the selective motor phenotype that is typical in SMA patients (reviewed in ref. ${ }^{6}$ ). In this regard, we have recently shown that, in Drosophila, perturbation of snRNP biogenesis factors pICln or Tgs1 causes motor deficits that mirror those brought about by loss of SMN or select Gemins including Gemin $3^{61}$. This corroborates earlier findings demonstrating that knockdown of pICln or U1 snRNP leads to MND-like phenotypes in zebrafish ${ }^{85,86}$. Notably, by discovering an interaction between Gemin 3 and either pICln or Tgs $1^{61}$, we underscored that these factors participate in a common pathway that most likely centres on the synthesis of snRNPs which form the backbone of the spliceosome. Here, we widen our findings by uncovering a genetic association between Gemin 3 and two RBPs with important roles in diverse 
A
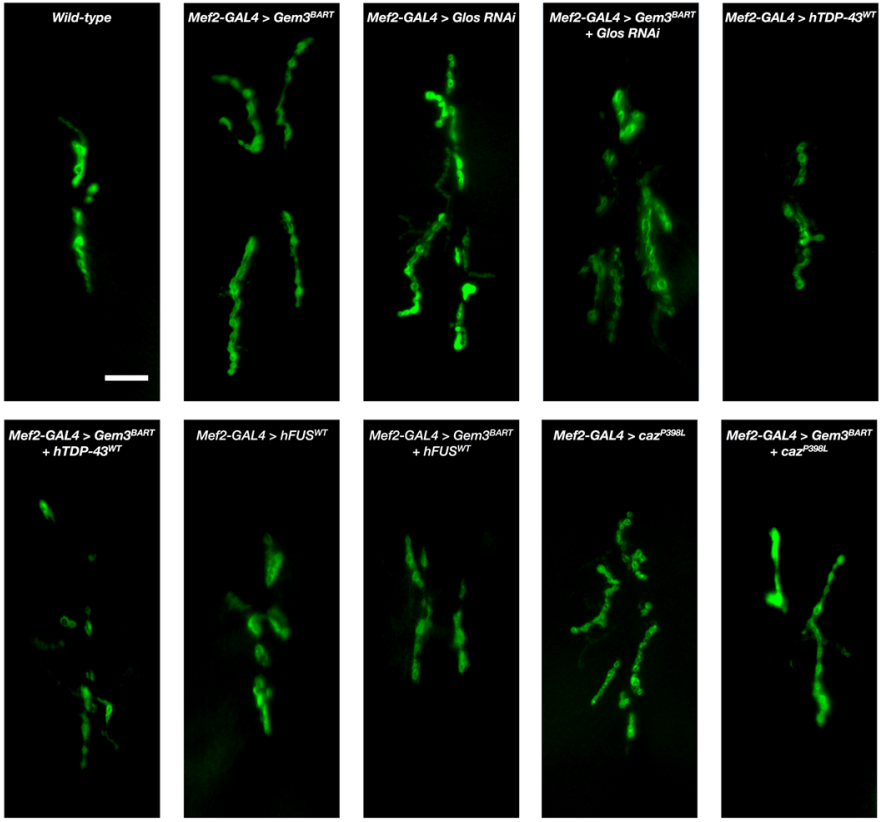

B

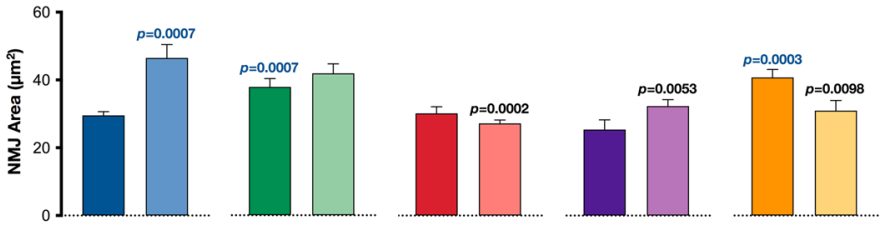

C

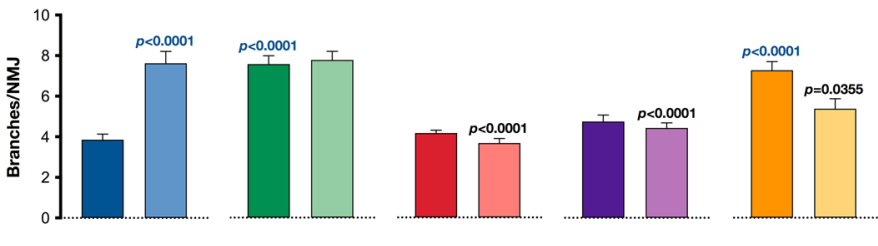

D

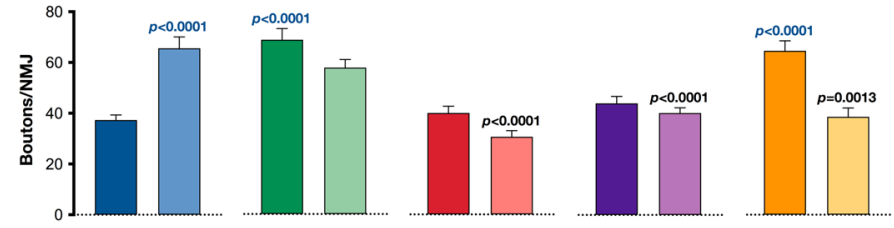

\begin{tabular}{|c|c|}
\hline Genotype & Bar \\
\hline Wild-type & $\square$ \\
\hline Mef2-GAL4 > Gem3 $3^{\text {AART }}$ & $\square$ \\
\hline Mef2-GAL4 > Glos RNAi & $\square$ \\
\hline Mef2-GAL4 > Gem3 $3^{B A R T}+$ Glos RNAi & $\square$ \\
\hline Mef2-GAL4 > hTDP- $43^{W T}$ & $\square$ \\
\hline Mef2-GAL4 > Gem3 $3^{B A R T}+h T D P-43^{W T}$ & $\square$ \\
\hline Mef2-GAL4 > hFUS ${ }^{w T}$ & $\square$ \\
\hline Mef2-GAL4 > Gem3 $3^{\text {BART }}+h F U S^{W T}$ & $\square$ \\
\hline Mef2-GAL4 > $\mathrm{Caz}^{\text {P398L }}$ & $\square$ \\
\hline Mef2-GAL4 > Gem3 $3^{B A A T}+\mathrm{caz}^{P 39 g L}$ & $\square$ \\
\hline
\end{tabular}

Figure 7. Overexpression of $h T D P-43$ or $h F U S / c a z^{P 398 L}$ suppresses NMJ expansion in Gem 3 mutant flies. (A) Representative images of NMJs innervating ventral longitudinal muscles 6 and 7 in third instar larvae stained with post-synaptic anti-DLG antibody $($ scale bar $=10 \mu \mathrm{m})$. Visual inspection reveals that compared to wild-type, muscle-directed expression of Gem $3^{\mathrm{BART}}$ induces an overgrown NMJ morphology that is restored on overexpression of $h T D P-43^{W T}, h F U S^{W T}$ and, to a lesser degree, $c a z^{P 398 L}$. An overgrowth phenotype is also obvious on Glos knockdown (applied singularly or combined with Gem3 ${ }^{\text {BART }}$ ) or in flies expressing $c a z^{P 398 L}$. These observations were confirmed upon quantification of NMJ area (B), number of branches per NMJ (C) and number of boutons within a single NMJ (D). In (B-D) data presented are the mean \pm S.E.M. and $n \geq 18$ per genotype. Equal number of male and female larvae were assessed. Significance as tested by the unpaired $t$-test is indicated by the exact $p$-value, shown either in blue (comparison to wild-type larvae) or black (comparison to Gem $3^{B A R T}$ larvae). 
A

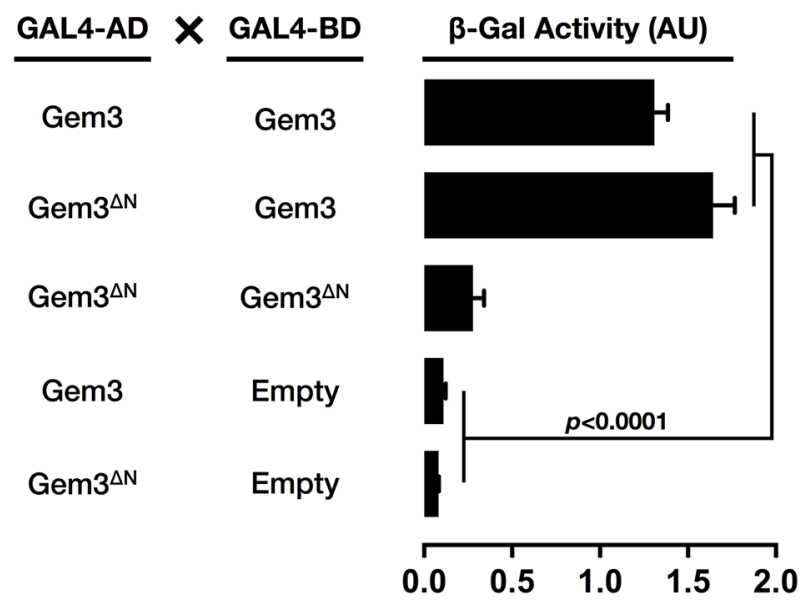

B

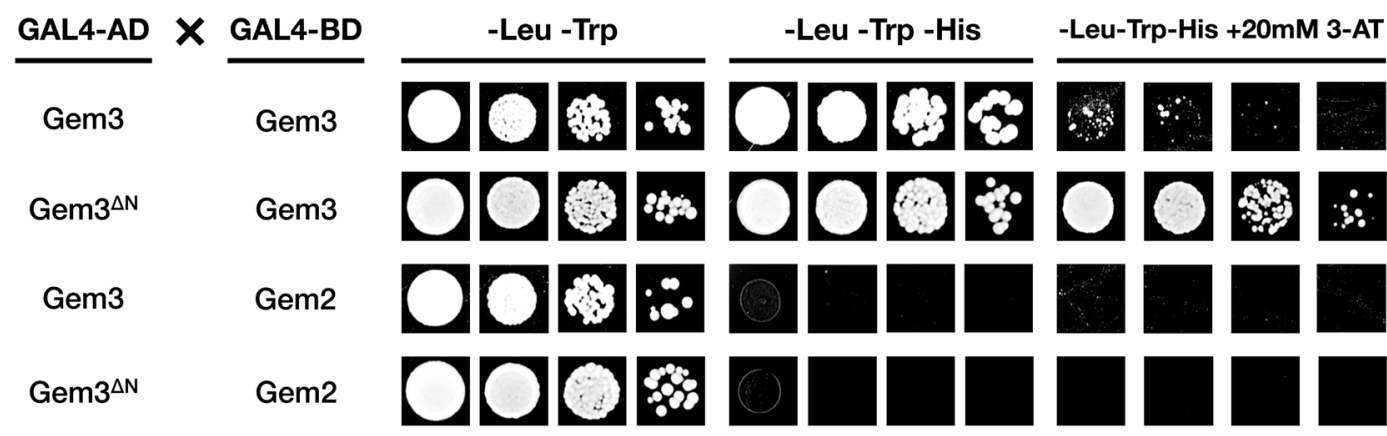

C

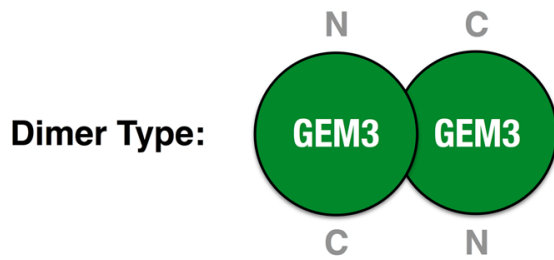

Gem3:Gem3

Homodimer

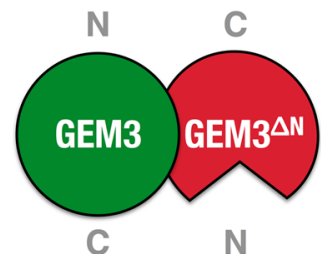

Gem3:Gem3sN

Heterodimer

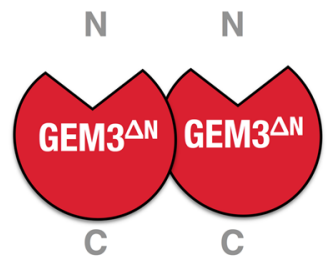

Gem3 $^{\Delta N}: G^{\prime} 3^{\Delta N}$

Homodimer

Favourability:

10

0000

\section{$x$}

Figure 8. Full-length Gemin 3 interacts not only with itself but also with $G e m 3^{\Delta N}$. (A) Measurement of $\beta$-galactosidase activity was used to assay the expression of the GAL1-LacZ reporter gene that is produced by the combinations of the indicated proteins. Full-length Gemin3 interacts with itself or with its N-terminal truncated form $\left(\mathrm{Gem} 3^{\Delta \mathrm{N}}\right)$. Gem $3^{\Delta \mathrm{N}}$ is not capable of self-binding. An empty vector served as a negative control. Individual bars represent the mean \pm S.E.M. $\beta$-galactosidase activity of 3 independent experiments. Significance as tested by one-way ANOVA is indicated by the exact $p$-value. (B) The reporter strain carrying yeast twohybrid plasmids expressing the indicated proteins was spotted in serial dilutions on -Leu-Trp-His plates in the presence of $20 \mathrm{mM} 3$-AT. Under these conditions, Gem3-Gem3 interaction is unfavourable whereas the Gem3-Gem $3^{\Delta \mathrm{N}}$ association remains favourable. Interaction between Gem 3 or Gem $3^{\Delta \mathrm{N}}$ with Gemin 2 served as a negative control. At least 3 independent experiments were performed, and the result of one representative is shown. (C) Model for Gemin3 dimerisation. Left, Gemin3 monomers are hypothesised to bind to each other in the reverse direction. Middle, The Gem $3^{\Delta \mathrm{N}}$ mutant is a truncated version of Gemin 3 lacking the N-terminus, which hosts the helicase domains. In the presence of $\mathrm{Gem}^{\Delta \mathrm{N}}$, wild-type:mutant dimers are favoured more than wild-type:wild-type dimers. Right, A model predicting self-association in the opposite orientation infers that Gem $3^{\Delta \mathrm{N}}$ is not self-binding, hence, mutant:mutant dimers do not form. Abbreviations: N, N-terminus; C, C-terminus. 
aspects of RNA metabolism, namely TDP-43 and FUS. Our data suggest that Gemin3, TDP-43 and FUS function in overlapping pathways that influence viability, muscle mass, NMJ morphology and motoric ability.

Considering the roles of Gemin3, TDP-43 and FUS at different points in the life of the spliceosome, we speculate that the intersecting pathways are vital for the correct splicing of mRNAs, which we believe is a main contributor to the health and optimal function of the neuromuscular system. In support, although its exact activities in snRNP assembly remain unclear, Gemin3 is indispensable for this process in vivo ${ }^{60}$. Considering TDP-43 and FUS, long before their implication in ALS, these two proteins were reported to influence pre-mRNA splicing or interact with known splicing factors ${ }^{87,88}$. Both RBPs were later shown to bind to predominantly UG-rich sequences in RNA transcripts, regulating the expression and alternative splicing of multiple yet distinct target genes, particularly those with exceptionally long introns ${ }^{89-93}$. Thus, loss of TDP-43 or FUS in cell lines or mouse brain leads to splicing defects that are mostly different for either factor ${ }^{89,90,92,94,95}$. Even splicing of snRNP components is altered based on studies in sporadic ALS patient-derived lower motor neurons that had nuclear TDP-43 depletion ${ }^{96}$ or FUS knockdown in a human cell line ${ }^{97}$. Overexpression of ALS-causing TDP-43 or FUS mutants, which cause neuromuscular phenotypes in mice, were also found to induce aberrant RNA splicing ${ }^{98,99}$. Similarly, Drosophila with knockout or overexpression of TBPH exhibit splicing alterations ${ }^{100}$. Interestingly, expression levels of several snRNAs were found significantly increased in brains of flies overexpressing human TDP-43 ${ }^{101}$ and decreased in fibroblasts derived from ALS patients with FUS mutations or FUS transgenic mice ${ }^{19}$. Notably, in an ALS mouse model, an endogenous C-terminal domain mutation in TDP-43 was recently reported to induce a gain of splicing function. Hence, splicing activity of TDP-43 was modified in such a way that it leads to the excision of otherwise normally conserved exons, thereby, termed 'skiptic exons' ${ }^{102}$. Pathogenic TDP-43 or FUS mutations are also known to affect splicing in a gain-of-toxic-function manner by mislocalising snRNPs, SMN and splicing factors (PSF and NeuN) to the cytosol ${ }^{18,19,86,103,104}$.

It is interesting to note that whereas gain of TDP-43 or FUS function were both shown to enhance Gemin3 motor deficits, we observed that loss-of-function was consequential only for FUS. This can be explained by the more intimate relationship of FUS with the SMN complex. In this respect, in addition to U1 (and U11) snRNP components $^{19,103}$, SMN complex members including SMN and Gemin3 were shown to be integral members of the FUS interactome ${ }^{19}$. It is plausible that the SMN complex might collaborate with FUS in an as yet unknown snRNP-related function, which is disrupted by deviations from normal Caz/FUS levels. Thus, ALS-causative mutations in FUS were found to strengthen the interaction with SMN potentially sequestering SMN and, most probably, its associates, from their normal localisation and function ${ }^{19}$. It is important to note that we do not exclude the possibility that Gemin 3 cooperates with TDP-43 and FUS in other steps of RNA metabolism including transcription or RNA transport given that all three factors are known to participate in either process ${ }^{11,12,28,60,105}$.

Gem3 mutant phenotypes were not hastened by SOD1 or C9orf72 gain-of-function. This allows us to infer that the genetic interaction between Gemin 3 and TBPH/TDP-43 or caz/FUS is specific. Nonetheless, reduced levels of Sod 1 brought about by heterozygosity for a Sod1 mutant or RNAi-mediated knockdown were surprisingly found to induce motor deficits in Gem 3 mutant flies during late adulthood. Oxidative stress is exacerbated by age ${ }^{106}$ and paucity of Sod $1^{107-109}$. Notably, snRNP assembly function of the SMN complex was found to be inhibited by oxidative stress in a dose-dependent manner ${ }^{110}$. This observation adds to the plethora of evidence showing that oxidative stress perturbs RNA metabolism (reviewed in ref. ${ }^{111}$ ). Thus, it is reasonable to speculate that oxidative stress is a modifying factor for Gemin3 function in snRNP synthesis as part of the SMN complex.

Ectopic overexpression of the helicase core deletion mutant Gem $3^{\Delta \mathrm{N}}$ in a wild-type background induces phenotypes that overlap those resulting from Gemin3 loss-of-function. In this regard, either overexpression of Gem $3^{\Delta \mathrm{N}}$ or RNAi-mediated knockdown of Gemin3, both targeted to muscle tissue, was shown previously to disrupt motor behaviour. Applied simultaneously, these two genetic manipulations were found to cause lethality ${ }^{64}$. Thus, the evidence favours the possibility that Gem $3^{\Delta \mathrm{N}}$ interferes with endogenous Gemin 3 to induce loss of function, hence acting as a dominant-negative mutant or a Muller's antimorph. Here, we show that Gemin3 is capable of self-interaction and $\mathrm{Gem} 3^{\Delta \mathrm{N}}$ retains the ability to interact with its wild-type counterpart. Importantly, analysis of interaction strength demonstrates that wild-type:mutant dimers are favoured more than wild-type:wild-type dimers (Fig. $8 \mathrm{C}$ ). Based on these findings, we predict that $\mathrm{Gem} 3^{\Delta \mathrm{N}}$ titres endogenous Gemin3 into non-functional dimers or oligomers. Sequestration of Gemin 3 can perturb SMN complex stoichiometry in addition to inhibiting the participation of Gemin3 in SMN complex-related activities including snRNP assembly or recycling.

The formation of wild-type:mutant dimers or even oligomers can potentially hinder the catalytic activity of Gemin3, thus raising the question of whether dimerization of Gemin3 is a prerequisite for its ATPase-dependent RNP chaperoning activities. Self-interaction that is independent of the RNA substrate is rather unusual for members of the DEAD-box RNA helicase family. It has been reported in prokaryotes for Escherichia coli RhIB $^{112}$, Thermus thermophilus Hera ${ }^{113}$, Bacillus subtilis $\mathrm{CshA}^{114}$ and cyanobacteria $\mathrm{CrhR}^{115}$. In this context, our findings add Gemin 3 to the growing list of eukaryotic DEAD-box RNA helicases that also have a self-interaction property including transcriptional regulators DDX5/p68 and DDX17/p $72^{116}$. Domain analysis of both T. thermophilus Hera and B. Subtilis CshA revealed that efficient dimerization is dependent on protein regions other than the those hosting the two highly-conserved RecA-like helicase domains which are required for RNA substrate binding and catalytic activity ${ }^{113,114}$. For E. coli RhIB, cyanobacteria CrhR ${ }^{115}$ and eukaryotic DDX5 or DDX17, a large part of the conserved core was required for self-association ${ }^{112,116}$, a finding that also applies for Gemin3. To this end, we show that, alone, the C-terminal domain of Gemin3 is incapable of self-binding, hence, the formation of Gem $3^{\Delta \mathrm{N}}$ homodimers is an unfavourable reaction (Fig. 8C). However, interaction is observed in the presence of the $\mathrm{N}$-terminus, making the formation of $\mathrm{Gem} 3^{\Delta \mathrm{N}}$ :Gem 3 dimers a highly favourable reaction. This indicates that self-interaction requires that the full-length protein is present in at least one of the two monomers, thus raising the possibility that Gemin3 monomers bind to each other in the reverse direction. This model warrants future investigation through further molecular and structural studies. 
Given the functional interaction of ALS-linked TBPH/TDP-43, Caz/FUS and Sod1 with Gemin3, which itself is intimately associated with the SMA-causative SMN, our work adds to the substantial collection of evidence supporting convergence of the molecular mechanisms of two major MNDs. Although we speculate that defects in RNA metabolism might be central to the pathophysiology of ALS and SMA, further investigation of the mechanistic overlaps is now possible in a genetically-tractable model organism. Importantly, given our findings, we propose Gemin3 as a candidate for modifying motor neuron degeneration.

\section{Materials and Methods}

Flies. Flies were cultured on food consisting of sugar, corn meal, yeast, and agar in plastic vials at an incubation temperature of $25^{\circ} \mathrm{C}$ under 12 hours day/night cycles. The wild-type strain was $w^{1118}$. For adult-based assays, male flies were used except where indicated. In instances where females were used, flies were virgins. For larval-based assays, equal number of male and female larvae were assessed. Inducible transgenes were expressed via the bipartite GAL4/upstream activation sequence (UAS) system (reviewed in ref. ${ }^{117}$ ). Muscle-exclusive expression was achieved through the use of the Mef2-GAL4 driver ${ }^{118}$. The Gem $3^{B A R T}$ allele (UAS.Gem $3^{\text {BART}}$ ) was generated previously by transposition of the Gem $3^{\Delta N}$ transgenic allele into a repressive region on chromosome $2^{68}$. The UAS.Glos$I R^{D E X}$ (Glos RNAi) transgene was described and characterised previously ${ }^{67} . T B P H^{\triangle 23}$ and $\mathrm{caz}^{1}$ are small deletions that partially remove the coding and 5' sequence of $T B P H^{73}$ and $c a z^{75}$, respectively. They are considered as null alleles of the respective gene. The Sod $1^{\text {G51S }}$ mutant (also known as Sod1 $1^{\text {n108 }}$ or Sod1 ${ }^{n 1}$ ) carries an EMS-generated missense mutation in the Sod 1 gene that disrupts dimer contact ${ }^{119}$. Sod $1^{\text {G51S }}$ homozygotes were reported to be null for superoxide dismutase activity. In Sod $1^{551 S}$ heterozygotes, superoxide dismutase activity was reported to be close to $40 \%{ }^{80}$. The RNAi transgenic constructs, UAS.TBPH-RNAi [Trinity] (ID: 38377), UAS.TBPH-RNAi [Merton] (ID: 38379), UAS.TBPH-RNAi [Maudlin] (ID: 104401), UAS.caz-RNAi [Kellogg] (ID: 100291), UAS.cazRNAi [Oriel] (ID: 330388), UAS.Sod1-RNAi [Hertford] (ID: 31551), and UAS.Sod1-RNAi [Pembroke] (ID: 108307) were obtained from the Vienna Drosophila Resource Center, Austria ${ }^{120}$, and were described previously ${ }^{73,121,122}$. The provenance of the various UAS transgenes for TBPH, hTDP-43, caz, hFUS, Sod 1 and $h S O D 1$ is referenced in Table 1. All C9orf72-related transgenic lines were obtained from the Bloomington Drosophila Stock Center (NIH P40OD018537) at Indiana University, USA and were characterised previously ${ }^{123}$. Combination of the various genetic tools including GAL4 drivers, alleles, and transgenes was performed according to standard genetic crossing schemes.

Mobility assays. Mobility assays in larvae and adult flies were conducted as described previously ${ }^{67}$. In brief, third instar larvae were first placed on a $0.7 \%$ agar plate. Subsequently, the number of forward body wall contractions exhibited by the organism in 30 seconds were counted. Each larva was assessed three times before an average was taken. To assess climbing performance in adult flies, two empty polystyrene tubes were vertically joined by tape facing each other. Flies (15-20) were then transferred into the lower tube and allowed to acclimatize. Flies were then gently tapped down to the bottom of the vial. The time for the first fly within a group to cross an $8 \mathrm{~cm}$ threshold was first measured. Consequently, the number of flies per group, that can climb above the $8 \mathrm{~cm}$ mark by 10 seconds, was determined. For each group of flies, four trials were performed. A minimum of four groups were assayed per genotype.

Flight assay. Flight performance was assessed as detailed previously ${ }^{61,67}$. This assay made use of the Droso-Drome apparatus, which consists of a $1 \mathrm{~L}$ glass bottle coated with an alcohol-based sticky fluid, and divided into 4 sectors, of $5 \mathrm{~cm}$ each, spanning a total height of $20 \mathrm{~cm}$. In short, flies first underwent a 'warm-up' by inducing negative geotaxis in an empty tube for 6 times. Organisms were then dropped into the Droso-Drome to induce flight. The number of flies stuck to each sector was next counted, divided by the total number of flies dropped and multiplied by 100 to generate the percentage number of flies per sector. Fight ability correlates with the height or sector in which flies are distributed, hence, fly percentages that are skewed towards the lower sectors of the Droso-Drome are indicative of reduced flight capacity.

Puparial axial ratios. Length and width of puparia were first measured from still images. As reported previously $^{61,67}$, calculation of puparial axial ratios involved dividing the length by the width of the puparia.

Immunohistochemistry. The same immunohistochemistry procedures described previously ${ }^{65}$ were followed. Briefly, body wall muscles of wandering third instar larvae were dissected in phosphate buffered saline (PBS), fixed in $4 \%$ paraformaldehyde in PBS and washed in PBS $+0.1 \%$ Triton X-100 (PBT). Tissues were then stained overnight at room temperature by mouse anti-Discs large antibody (1:1000; Developmental Studies Hybridoma Bank, University of Iowa, USA). On the following day, tissues were washed in PBT and stained overnight at room temperature with anti-mouse Alexa Fluor 488-conjugated secondary goat antibody (1:50) and Alexa Fluor 546-conjugated Phalloidin (1:50). After a final wash in PBT, the samples were mounted in $90 \%$ glycerol with anti-fade. Imaging was performed with Optika B-600TiFL microscope (20x or 40x objectives).

Analysis of muscle size and NMJ morphology. ImageJ software (NIH) was used to quantify both muscle and NMJ area. The former comprised of both ventral longitudinal muscles 6 and 7 derived from abdominal segments 2-4 whereas the latter constituted the postsynaptic region on the same muscles stained by the anti-Discs large antibody. Branch number was determined by counting the number of arborisations containing at least two boutons within a single NMJ. To determine, bouton numbers, all boutons were counted within a single NMJ.

Yeast two-hybrid assays. Two-hybrid assays were performed as described previously ${ }^{67}$. Briefly, baits and preys were obtained by PCR amplification of cDNA and ligation into the $p A S \Delta \Delta$ and $p A C T 2 s t$ vectors, respectively ${ }^{124}$. Primer sequences and PCR regimes are available upon request. The cDNA clones for Gemin2 (LD47479) 
and Gemin3 (LD05563) were obtained from the Drosophila Genomics Resource Centre (Indiana University, USA). Gem $3^{\Delta N}$ was synthesised as described previously ${ }^{65}$. The bait $p A S \Delta \Delta$ construct containing the protein sequence fused in frame with the GAL4 DNA binding domain (GAL4-BD) was used to transform the CG1945 strain, which was then selected on -Trp plates. The prey $p A C T 2 s t$ construct containing the protein sequence fused in frame with the GAL4 activation domain (GAL4-AD) was used to transform the Y187 strain, which was then selected on -Leu plates. Mating of bait and prey strains was achieved overnight on rich yeast extract peptone dextrose (YPD) plates and - Trp -Leu plates were used to select diploids containing bait/prey combinations. Protein-protein interactions were screened by spotting serial dilutions on -Trp-Leu-His plates. Where indicated, $30 \mathrm{mM}$ 3-amino-1,2,4-triazole (3-AT) was added to the medium to assess interaction strength. Incubations were performed at $28^{\circ} \mathrm{C}$ for 3 to 5 days. The $\beta$-galactosidase assay was used to quantify yeast two-hybrid interactions. Cells were grown in -Trp-Leu selective medium to an $\mathrm{OD}_{600}=0.5-1.0$. Activity was measured from extracts as reported previously ${ }^{125}$.

Statistical analysis. Values are presented as means \pm S.E.M. unless otherwise indicated. The unpaired $t$-test was used to compare measures between 2 groups whereas one-way ANOVA was applied for multiple comparisons with the control. Two-way ANOVA, followed by Bonferroni's post hoc test, was used to determine differences between 2 groups in the percentage number of fliers (sectors 2-4) vs. non-fliers (sector 1) in the flight assay (GraphPad Prism v8.0.1). Differences were deemed statistically significant if $p<0.05$, and when this is the case, the exact $p$-value is presented.

\section{Data availability}

All data generated or analysed during this study are included in this published article.

Received: 12 March 2019; Accepted: 29 October 2019;

Published online: 10 December 2019

\section{References}

1. Ibrahim, F., Nakaya, T. \& Mourelatos, Z. RNA dysregulation in diseases of motor neurons. Annu Rev Pathol 7, 323-352, https://doi. org/10.1146/annurev-pathol-011110-130307 (2012).

2. Baumer, D., Ansorge, O., Almeida, M. \& Talbot, K. The role of RNA processing in the pathogenesis of motor neuron degeneration. Expert reviews in molecular medicine 12, e21, https://doi.org/10.1017/S1462399410001523 (2010).

3. Ito, D., Hatano, M. \& Suzuki, N. RNA binding proteins and the pathological cascade in ALS/FTD neurodegeneration. Sci Transl Med 9, https://doi.org/10.1126/scitranslmed.aah5436 (2017).

4. Butti, Z. \& Patten, S. A. RNA Dysregulation in Amyotrophic Lateral Sclerosis. Front Genet 9, 712, https://doi.org/10.3389/ fgene.2018.00712 (2018).

5. Burghes, A. H. \& Beattie, C. E. Spinal muscular atrophy: why do low levels of survival motor neuron protein make motor neurons sick? Nat Rev Neurosci 10, 597-609 (2009).

6. Lanfranco, M., Vassallo, N. \& Cauchi, R. J. Spinal Muscular Atrophy: From Defective Chaperoning of snRNP Assembly to Neuromuscular Dysfunction. Front Mol Biosci 4, 41, https://doi.org/10.3389/fmolb.2017.00041 (2017).

7. Cauchi, R. J. S. M. N. and Gemins: 'we are family'... or are we? Insights into the partnership between Gemins and the spinal muscular atrophy disease protein SMN. Bioessays 32, 1077-1089 (2010).

8. Donlin-Asp, P. G., Bassell, G. J. \& Rossoll, W. A role for the survival of motor neuron protein in mRNP assembly and transport. Curr Opin Neurobiol 39, 53-61, https://doi.org/10.1016/j.conb.2016.04.004 (2016).

9. Brown, R. H. \& Al-Chalabi, A. Amyotrophic Lateral Sclerosis. N Engl J Med 377, 162-172, https://doi.org/10.1056/NEJMra1603471 (2017).

10. Taylor, J. P., Brown, R. H. Jr. \& Cleveland, D. W. Decoding ALS: from genes to mechanism. Nature 539, 197-206, https://doi. org/10.1038/nature20413 (2016).

11. Lagier-Tourenne, C., Polymenidou, M. \& Cleveland, D. W. TDP-43 and FUS/TLS: emerging roles in RNA processing and neurodegeneration. Hum Mol Genet 19, R46-64, https://doi.org/10.1093/hmg/ddq137 (2010).

12. Ratti, A. \& Buratti, E. Physiological functions and pathobiology of TDP-43 and FUS/TLS proteins. J Neurochem 138(Suppl 1), 95-111, https://doi.org/10.1111/jnc.13625 (2016).

13. Turner, B. J., Parkinson, N. J., Davies, K. E. \& Talbot, K. Survival motor neuron deficiency enhances progression in an amyotrophic lateral sclerosis mouse model. Neurobiol Dis 34, 511-517, https://doi.org/10.1016/j.nbd.2009.03.005 (2009).

14. Wang, I. F., Reddy, N. M. \& Shen, C. K. Higher order arrangement of the eukaryotic nuclear bodies. Proc Natl Acad Sci USA 99, 13583-13588, https://doi.org/10.1073/pnas.212483099 (2002).

15. Tsuiji, H. et al. Spliceosome integrity is defective in the motor neuron diseases ALS and SMA. EMBO Mol Med 5, 221-234, https:// doi.org/10.1002/emmm.201202303 (2013).

16. Yamazaki, T. et al. FUS-SMN protein interactions link the motor neuron diseases ALS and SMA. Cell Rep 2, 799-806, https://doi. org/10.1016/j.celrep.2012.08.025 (2012).

17. Groen, E. J. et al. ALS-associated mutations in FUS disrupt the axonal distribution and function of SMN. Hum Mol Genet 22, 3690-3704, https://doi.org/10.1093/hmg/ddt222 (2013).

18. Gerbino, V., Carri, M. T., Cozzolino, M., Achsel, T. \& Mislocalised, F. U. S. mutants stall spliceosomal snRNPs in the cytoplasm. Neurobiol Dis 55, 120-128, https://doi.org/10.1016/j.nbd.2013.03.003 (2013).

19. Sun, S. et al. ALS-causative mutations in FUS/TLS confer gain and loss of function by altered association with SMN and U1-snRNP. Nat Commun 6, 6171, https://doi.org/10.1038/ncomms7171 (2015).

20. Yin, S. et al. Evidence that C9ORF72 Dipeptide Repeat Proteins Associate with U2 snRNP to Cause Mis-splicing in ALS/FTD Patients. Cell Rep 19, 2244-2256, https://doi.org/10.1016/j.celrep.2017.05.056 (2017).

21. Liu, Q. \& Dreyfuss, G. A novel nuclear structure containing the survival of motor neurons protein. Embo J 15, 3555-3565 (1996).

22. Cauchi, R. J. Gem formation upon constitutive Gemin3 overexpression in Drosophila. Cell Biol Int 35, 1233-1238, https://doi. org/10.1042/CBI20110147 (2011).

23. Mirra, A. et al. Functional interaction between FUS and SMN underlies SMA-like splicing changes in wild-type hFUS mice. Sci Rep 7, 2033, https://doi.org/10.1038/s41598-017-02195-0 (2017).

24. Ishihara, T. et al. Decreased number of Gemini of coiled bodies and U12 snRNA level in amyotrophic lateral sclerosis. Hum Mol Genet 22, 4136-4147, https://doi.org/10.1093/hmg/ddt262 (2013).

25. Workman, E., Kolb, S. J. \& Battle, D. J. Spliceosomal small nuclear ribonucleoprotein biogenesis defects and motor neuron selectivity in spinal muscular atrophy. Brain Res 1462, 93-99, https://doi.org/10.1016/j.brainres.2012.02.051 (2012). 
26. Zhang, Z. et al. Dysregulation of synaptogenesis genes antecedes motor neuron pathology in spinal muscular atrophy. Proc Natl Acad Sci USA 110, 19348-19353, https://doi.org/10.1073/pnas.1319280110 (2013).

27. Boulisfane, N. et al. Impaired minor tri-snRNP assembly generates differential splicing defects of U12-type introns in lymphoblasts derived from a type I SMA patient. Hum Mol Genet 20, 641-648, https://doi.org/10.1093/hmg/ddq508 (2011).

28. Yasuda, K. \& Mili, S. Dysregulated axonal RNA translation in amyotrophic lateral sclerosis. Wiley Interdiscip Rev RNA 7, 589-603, https://doi.org/10.1002/wrna.1352 (2016).

29. Fallini, C., Bassell, G. J. \& Rossoll, W. Spinal muscular atrophy: the role of SMN in axonal mRNA regulation. Brain Res 1462, 81-92, https://doi.org/10.1016/j.brainres.2012.01.044 (2012).

30. Baldwin, K. R., Godena, V. K., Hewitt, V. L. \& Whitworth, A. J. Axonal transport defects are a common phenotype in Drosophila models of ALS. Hum Mol Genet 25, 2378-2392, https://doi.org/10.1093/hmg/ddw105 (2016).

31. Corcia, P. et al. Phenotypic and genotypic studies of ALS cases in ALS-SMA families. Amyotroph Lateral Scler Frontotemporal Degener 19, 432-437, https://doi.org/10.1080/21678421.2018.1440406 (2018).

32. Blauw, H. M. et al. SMN1 gene duplications are associated with sporadic ALS. Neurology 78, 776-780, https://doi.org/10.1212/ WNL.0b013e318249f697 (2012)

33. Corcia, P. et al. SMN1 gene, but not SMN2, is a risk factor for sporadic ALS. Neurology 67, 1147-1150, https://doi.org/10.1212/01. wnl.0000233830.85206.1e (2006)

34. Veldink, J. H. et al. SMN genotypes producing less SMN protein increase susceptibility to and severity of sporadic ALS. Neurology 65, 820-825, https://doi.org/10.1212/01.wnl.0000174472.03292.dd (2005).

35. Cauchi, R. J. Gem depletion: amyotrophic lateral sclerosis and spinal muscular atrophy crossover. CNS Neurosci Ther 20, 574-581, https://doi.org/10.1111/cns.12242 (2014).

36. Rodriguez-Muela, N. et al. Single-Cell Analysis of SMN Reveals Its Broader Role in Neuromuscular Disease. Cell Rep 18, 1484-1498, https://doi.org/10.1016/j.celrep.2017.01.035 (2017).

37. Mercuri, E. et al. Nusinersen versus Sham Control in Later-Onset Spinal Muscular Atrophy. N Engl J Med 378, 625-635, https:// doi.org/10.1056/NEJMoa1710504 (2018).

38. Finkel, R. S. et al. Nusinersen versus Sham Control in Infantile-Onset Spinal Muscular Atrophy. N Engl J Med 377, 1723-1732, https://doi.org/10.1056/NEJMoa1702752 (2017).

39. Turner, B. J. et al. Overexpression of survival motor neuron improves neuromuscular function and motor neuron survival in mutant SOD1 mice. Neurobiol Aging 35, 906-915, https://doi.org/10.1016/j.neurobiolaging.2013.09.030 (2013).

40. Kariya, S. et al. Mutant superoxide dismutase 1 (SOD1), a cause of amyotrophic lateral sclerosis, disrupts the recruitment of SMN, the spinal muscular atrophy protein to nuclear Cajal bodies. Hum Mol Genet 21, 3421-3434, https://doi.org/10.1093/hmg/dds174 (2012).

41. Perera, N. D. et al. Enhancing survival motor neuron expression extends lifespan and attenuates neurodegeneration in mutant TDP-43 mice. Hum Mol Genet 25, 4080-4093, https://doi.org/10.1093/hmg/ddw247 (2016).

42. Aquilina, B. \& Cauchi, R. J. Modelling motor neuron disease in fruit flies: Lessons from spinal muscular atrophy. J Neurosci Methods, https://doi.org/10.1016/j.jneumeth.2018.04.003 (2018).

43. Olesnicky, E. C. \& Wright, E. G. Drosophila as a Model for Assessing the Function of RNA-Binding Proteins during Neurogenesis and Neurological Disease. J Dev Biol 6, https://doi.org/10.3390/jdb6030021 (2018).

44. Sen, A. et al. Genetic circuitry of Survival motor neuron, the gene underlying spinal muscular atrophy. Proc Natl Acad Sci USA 110, E2371-2380, https://doi.org/10.1073/pnas.1301738110 (2013).

45. Chang, H. C. et al. Modeling spinal muscular atrophy in Drosophila. PLoS ONE 3, e3209 (2008).

46. Aquilina, B. \& Cauchi, R. J. Genetic screen identifies a requirement for SMN in mRNA localisation within the Drosophila oocyte. BMC Res Notes 11, 378, https://doi.org/10.1186/s13104-018-3496-1 (2018).

47. Dimitriadi, M. et al. Conserved genes act as modifiers of invertebrate SMN loss of function defects. PLoS Genet 6, e1001172, https://doi.org/10.1371/journal.pgen.1001172 (2010).

48. Freibaum, B. D. et al. GGGGCC repeat expansion in C9orf72 compromises nucleocytoplasmic transport. Nature 525, 129-133, https://doi.org/10.1038/nature14974 (2015).

49. Zhang, K. et al. The C9orf72 repeat expansion disrupts nucleocytoplasmic transport. Nature 525, 56-61, https://doi.org/10.1038/ nature14973 (2015).

50. Casci, I. \& Pandey, U. B. A fruitful endeavor: modeling ALS in the fruit fly. Brain Res 1607, 47-74, https://doi.org/10.1016/j. brainres.2014.09.064 (2015)

51. Steyaert, J. et al. FUS-induced neurotoxicity in Drosophila is prevented by downregulating nucleocytoplasmic transport proteins. Hum Mol Genet 27, 4103-4116, https://doi.org/10.1093/hmg/ddy303 (2018).

52. Zhan, L., Hanson, K. A., Kim, S. H., Tare, A. \& Tibbetts, R. S. Identification of genetic modifiers of TDP-43 neurotoxicity in Drosophila. PLoS One 8, e57214, https://doi.org/10.1371/journal.pone.0057214 (2013).

53. Borg, R. \& Cauchi, R. J. GEMINs: potential therapeutic targets for spinal muscular atrophy? Front Neurosci 8, 325, https://doi. org/10.3389/fnins.2014.00325 (2014).

54. Hua, Y. et al. Motor neuron cell-nonautonomous rescue of spinal muscular atrophy phenotypes in mild and severe transgenic mouse models. Genes \& development 29, 288-297, https://doi.org/10.1101/gad.256644.114 (2015).

55. Lee, A. J., Awano, T., Park, G. H. \& Monani, U. R. Limited phenotypic effects of selectively augmenting the SMN protein in the neurons of a mouse model of severe spinal muscular atrophy. PloS one 7, e46353, https://doi.org/10.1371/journal.pone.0046353 (2012).

56. Boyer, J. G., Ferrier, A. \& Kothary, R. More than a bystander: the contributions of intrinsic skeletal muscle defects in motor neuron diseases. Front Physiol 4, 356, https://doi.org/10.3389/fphys.2013.00356 (2013)

57. Loeffler, J. P., Picchiarelli, G., Dupuis, L. \& Gonzalez De Aguilar, J. L. The Role of Skeletal Muscle in Amyotrophic Lateral Sclerosis. Brain Pathol 26, 227-236, https://doi.org/10.1111/bpa.12350 (2016).

58. Hamilton, G. \& Gillingwater, T. H. Spinal muscular atrophy: going beyond the motor neuron. Trends Mol Med 19, 40-50, https:// doi.org/10.1016/j.molmed.2012.11.002 (2013).

59. Nash, L. A., Burns, J. K., Chardon, J. W., Kothary, R. \& Parks, R. J. Spinal Muscular Atrophy: More than a Disease of Motor Neurons? Curr Mol Med 16, 779-792, https://doi.org/10.2174/1566524016666161128113338 (2016).

60. Curmi, F. \& Cauchi, R. J. The multiple lives of DEAD-box RNA helicase DP103/DDX20/Gemin3. Biochem Soc Trans 46, 329-341, https://doi.org/10.1042/BST20180016 (2018).

61. Borg, R. M., Fenech Salerno, B., Vassallo, N., Bordonne, R. \& Cauchi, R. J. Disruption of snRNP biogenesis factors Tgs1 and pICln induces phenotypes that mirror aspects of SMN-Gemins complex perturbation in Drosophila, providing new insights into spinal muscular atrophy. Neurobiol Dis 94, 245-258, https://doi.org/10.1016/j.nbd.2016.06.015 (2016).

62. Chan, Y. B. et al. Neuromuscular defects in a Drosophila survival motor neuron gene mutant. Hum. Mol. Genet. 12, 1367-1376 (2003).

63. Rajendra, T. K. et al. A Drosophila melanogaster model of spinal muscular atrophy reveals a function for SMN in striated muscle. JCell Biol 176, 831-841 (2007).

64. Borg, R. \& Cauchi, R. J. The Gemin Associates of Survival Motor Neuron are Required for Motor Function in Drosophila. PLoS ONE 8, e83878 (2013).

65. Cauchi, R. J., Davies, K. E. \& Liu, J. L. A motor function for the DEAD-box RNA helicase, Gemin3, in Drosophila. PLoS Genet 4 , e1000265 (2008) 
66. Shpargel, K. B., Praveen, K., Rajendra, T. K. \& Matera, A. G. Gemin3 is an essential gene required for larval motor function and pupation in Drosophila. Mol Biol Cell 20, 90-101 (2009).

67. Lanfranco, M. et al. Novel interactors of the Drosophila Survival Motor Neuron (SMN) Complex suggest its full conservation. FEBS Lett 591, 3600-3614, https://doi.org/10.1002/1873-3468.12853 (2017).

68. Borg, R. M., Bordonne, R., Vassallo, N. \& Cauchi, R. J. Genetic Interactions between the Members of the SMN-Gemins Complex in Drosophila. PloS one 10, e0130974, https://doi.org/10.1371/journal.pone.0130974 (2015).

69. Neumann, M. et al. Ubiquitinated TDP-43 in frontotemporal lobar degeneration and amyotrophic lateral sclerosis. Science 314, 130-133, https://doi.org/10.1126/science.1134108 (2006).

70. Arai, T. et al. TDP-43 is a component of ubiquitin-positive tau-negative inclusions in frontotemporal lobar degeneration and amyotrophic lateral sclerosis. Biochem Biophys Res Commun 351, 602-611, https://doi.org/10.1016/j.bbrc.2006.10.093 (2006).

71. Lee, E. B., Lee, V. M. \& Trojanowski, J. Q. Gains or losses: molecular mechanisms of TDP43-mediated neurodegeneration. Nat Rev Neurosci 13, 38-50, https://doi.org/10.1038/nrn3121 (2011).

72. Voigt, A. et al. TDP-43-mediated neuron loss in vivo requires RNA-binding activity. PLoS One 5, e12247, https://doi.org/10.1371/ journal.pone.0012247 (2010).

73. Feiguin, F. et al. Depletion of TDP-43 affects Drosophila motoneurons terminal synapsis and locomotive behavior. FEBS Lett 583, 1586-1592, https://doi.org/10.1016/j.febslet.2009.04.019 (2009).

74. Ederle, H. \& Dormann, D. TDP-43 and FUS en route from the nucleus to the cytoplasm. FEBS Lett 591, 1489-1507, https://doi. org/10.1002/1873-3468.12646 (2017).

75. Wang, J. W., Brent, J. R., Tomlinson, A., Shneider, N. A. \& McCabe, B. D. The ALS-associated proteins FUS and TDP-43 function together to affect Drosophila locomotion and life span. J Clin Invest 121, 4118-4126, https://doi.org/10.1172/JCI57883 (2011).

76. Lanson, N. A. Jr. et al. A Drosophila model of FUS-related neurodegeneration reveals genetic interaction between FUS and TDP43. Hum Mol Genet 20, 2510-2523, https://doi.org/10.1093/hmg/ddr150 (2011).

77. Deng, H. X. et al. FUS-immunoreactive inclusions are a common feature in sporadic and non-SOD1 familial amyotrophic lateral sclerosis. Ann Neurol 67, 739-748, https://doi.org/10.1002/ana.22051 (2010).

78. Mizielinska, S. et al. C9orf72 repeat expansions cause neurodegeneration in Drosophila through arginine-rich proteins. Science 345, 1192-1194, https://doi.org/10.1126/science.1256800 (2014).

79. Pasinelli, P. \& Brown, R. H. Molecular biology of amyotrophic lateral sclerosis: insights from genetics. Nat Rev Neurosci 7, 710-723, https://doi.org/10.1038/nrn1971 (2006).

80. Phillips, J. P. et al. Subunit-destabilizing mutations in Drosophila copper/zinc superoxide dismutase: neuropathology and a model of dimer dysequilibrium. Proc Natl Acad Sci USA 92, 8574-8578 (1995).

81. Matera, A. G. et al. Composition of the Survival Motor Neuron (SMN) Complex in Drosophila melanogaster. G3 (Bethesda) 9 , 491-503, https://doi.org/10.1534/g3.118.200874 (2019).

82. Charroux, B. et al. Gemin4: a novel component of the SMN complex that is found in both gems and nucleoli. J. Cell Biol. 148, $1177-1186(2000)$

83. Hilton, J. L., Kearney, P. C. \& Ames, B. N. Mode of action of the herbicide, 3-amino-1,2,4-triazole(amitrole): inhibition of an enzyme of histidine biosynthesis. Arch Biochem Biophys 112, 544-547 (1965).

84. Klopotowski, T. \& Wiater, A. Synergism of aminotriazole and phosphate on the inhibition of yeast imidazole glycerol phosphate dehydratase. Arch Biochem Biophys 112, 562-566 (1965).

85. Winkler, C. et al. Reduced U snRNP assembly causes motor axon degeneration in an animal model for spinal muscular atrophy. Genes Dev. 19, 2320-2330 (2005).

86. Yu, Y. et al. U1 snRNP is mislocalized in ALS patient fibroblasts bearing NLS mutations in FUS and is required for motor neuron outgrowth in zebrafish. Nucleic Acids Res 43, 3208-3218, https://doi.org/10.1093/nar/gkv157 (2015).

87. Yang, L., Embree, L. J., Tsai, S. \& Hickstein, D. D. Oncoprotein TLS interacts with serine-arginine proteins involved in RNA splicing. J Biol Chem 273, 27761-27764 (1998).

88. Buratti, E. et al. Nuclear factor TDP-43 and SR proteins promote in vitro and in vivo CFTR exon 9 skipping. EMBO J 20, 1774-1784, https://doi.org/10.1093/emboj/20.7.1774 (2001).

89. Lagier-Tourenne, C. et al. Divergent roles of ALS-linked proteins FUS/TLS and TDP-43 intersect in processing long pre-mRNAs. Nat Neurosci 15, 1488-1497, https://doi.org/10.1038/nn.3230 (2012).

90. Polymenidou, M. et al. Long pre-mRNA depletion and RNA missplicing contribute to neuronal vulnerability from loss of TDP-43. Nat Neurosci 14, 459-468, https://doi.org/10.1038/nn.2779 (2011).

91. Tollervey, J. R. et al. Characterizing the RNA targets and position-dependent splicing regulation by TDP-43. Nat Neurosci 14, 452-458, https://doi.org/10.1038/nn.2778 (2011).

92. Rogelj, B. et al. Widespread binding of FUS along nascent RNA regulates alternative splicing in the brain. Sci Rep 2, 603, https:// doi.org/10.1038/srep00603 (2012).

93. Colombrita, C. et al. TDP-43 and FUS RNA-binding proteins bind distinct sets of cytoplasmic messenger RNAs and differently regulate their post-transcriptional fate in motoneuron-like cells. J Biol Chem 287, 15635-15647, https://doi.org/10.1074/jbc. M111.333450 (2012).

94. Colombrita, C. et al. From transcriptomic to protein level changes in TDP-43 and FUS loss-of-function cell models. Biochim Biophys Acta 1849, 1398-1410, https://doi.org/10.1016/j.bbagrm.2015.10.015 (2015).

95. Ling, J. P., Pletnikova, O., Troncoso, J. C. \& Wong, P. C. TDP-43 repression of nonconserved cryptic exons is compromised in ALSFTD. Science 349, 650-655, https://doi.org/10.1126/science.aab0983 (2015)

96. Highley, J. R. et al. Loss of nuclear TDP-43 in amyotrophic lateral sclerosis (ALS) causes altered expression of splicing machinery and widespread dysregulation of RNA splicing in motor neurones. Neuropathol Appl Neurobiol 40, 670-685, https://doi. org/10.1111/nan.12148 (2014).

97. van Blitterswijk, M. et al. Characterization of FUS mutations in amyotrophic lateral sclerosis using RNA-Seq. PLoS One 8, e60788, https://doi.org/10.1371/journal.pone.0060788 (2013).

98. Arnold, E. S. et al. ALS-linked TDP-43 mutations produce aberrant RNA splicing and adult-onset motor neuron disease without aggregation or loss of nuclear TDP-43. Proc Natl Acad Sci USA 110, E736-745, https://doi.org/10.1073/pnas.1222809110 (2013).

99. Qiu, H. et al. ALS-associated mutation FUS-R521C causes DNA damage and RNA splicing defects. JClin Invest 124, 981-999, https://doi.org/10.1172/JCI72723 (2014).

100. Hazelett, D. J., Chang, J. C., Lakeland, D. L. \& Morton, D. B. Comparison of parallel high-throughput RNA sequencing between knockout of TDP-43 and its overexpression reveals primarily nonreciprocal and nonoverlapping gene expression changes in the central nervous system of Drosophila. G3 (Bethesda) 2, 789-802, https://doi.org/10.1534/g3.112.002998 (2012).

101. Chung, C. Y. et al. Aberrant activation of non-coding RNA targets of transcriptional elongation complexes contributes to TDP-43 toxicity. Nat Commun 9, 4406, https://doi.org/10.1038/s41467-018-06543-0 (2018).

102. Fratta, P. et al. Mice with endogenous TDP-43 mutations exhibit gain of splicing function and characteristics of amyotrophic lateral sclerosis. EMBO J 37, https://doi.org/10.15252/embj.201798684 (2018).

103. Reber, S. et al. Minor intron splicing is regulated by FUS and affected by ALS-associated FUS mutants. EMBO J 35, 1504-1521, https://doi.org/10.15252/embj.201593791 (2016).

104. Wang, G. et al. Cytoplasmic mislocalization of RNA splicing factors and aberrant neuronal gene splicing in TDP-43 transgenic pig brain. Mol Neurodegener 10, 42, https://doi.org/10.1186/s13024-015-0036-5 (2015). 
105. Coyne, A. N., Zaepfel, B. L. \& Zarnescu, D. C. Failure to Deliver and Translate-New Insights into RNA Dysregulation in ALS. Front Cell Neurosci 11, 243, https://doi.org/10.3389/fncel.2017.00243 (2017).

106. Oka, S., Hirai, J., Yasukawa, T., Nakahara, Y. \& Inoue, Y. H. A correlation of reactive oxygen species accumulation by depletion of superoxide dismutases with age-dependent impairment in the nervous system and muscles of Drosophila adults. Biogerontology 16, 485-501, https://doi.org/10.1007/s10522-015-9570-3 (2015).

107. Sahin, A. et al. Human SOD1 ALS Mutations in a Drosophila Knock-In Model Cause Severe Phenotypes and Reveal DosageSensitive Gain- and Loss-of-Function Components. Genetics 205, 707-723, https://doi.org/10.1534/genetics.116.190850 (2017).

108. Phillips, J. P., Campbell, S. D., Michaud, D., Charbonneau, M. \& Hilliker, A. J. Null mutation of copper/zinc superoxide dismutase in Drosophila confers hypersensitivity to paraquat and reduced longevity. Proc Natl Acad Sci USA 86, 2761-2765, https://doi. org/10.1073/pnas.86.8.2761 (1989).

109. Parkes, T. L., Kirby, K., Phillips, J. P. \& Hilliker, A. J. Transgenic analysis of the cSOD-null phenotypic syndrome in Drosophila. Genome 41, 642-651 (1998).

110. Wan, L., Ottinger, E., Cho, S. \& Dreyfuss, G. Inactivation of the SMN complex by oxidative stress. Mol Cell 31, 244-254, https://doi. org/10.1016/j.molcel.2008.06.004 (2008).

111. Bozzo, F., Mirra, A. \& Carri, M. T. Oxidative stress and mitochondrial damage in the pathogenesis of ALS: New perspectives. Neurosci Lett 636, 3-8, https://doi.org/10.1016/j.neulet.2016.04.065 (2017).

112. Liou, G. G., Chang, H. Y., Lin, C. S. \& Lin-Chao, S. DEAD box RhlB RNA helicase physically associates with exoribonuclease PNPase to degrade double-stranded RNA independent of the degradosome-assembling region of RNase E. J Biol Chem 277, 41157-41162, https://doi.org/10.1074/jbc.M206618200 (2002).

113. Klostermeier, D. \& Rudolph, M. G. A novel dimerization motif in the C-terminal domain of the Thermus thermophilus DEAD box helicase Hera confers substantial flexibility. Nucleic Acids Res 37, 421-430, https://doi.org/10.1093/nar/gkn947 (2009).

114. Lehnik-Habrink, M. et al. The RNA degradosome in Bacillus subtilis: identification of CshA as the major RNA helicase in the multiprotein complex. Mol Microbiol 77, 958-971, https://doi.org/10.1111/j.1365-2958.2010.07264.x (2010).

115. Skeik, R. M. MSc Thesis: Dimerization of the DEAD-Box Cyanobacterial RNA Helicase Redox, CrhR Master of Science thesis, University of Alberta (2012).

116. Ogilvie, V. C. et al. The highly related DEAD box RNA helicases $\mathrm{p} 68$ and p72 exist as heterodimers in cells. Nucleic Acids Res 31, 1470-1480 (2003).

117. Cauchi, R. J. \& van den Heuvel, M. The fly as a model for neurodegenerative diseases: is it worth the jump? Neurodegener Dis 3 , 338-356 (2006)

118. Ranganayakulu, G., Schulz, R. A. \& Olson, E. N. Wingless signaling induces nautilus expression in the ventral mesoderm of the Drosophila embryo. Dev Biol 176, 143-148 (1996).

119. Campbell, S. D., Hilliker, A. J. \& Phillips, J. P. Cytogenetic analysis of the cSOD microregion in Drosophila melanogaster. Genetics 112, 205-215 (1986).

120. Dietzl, G. et al. A genome-wide transgenic RNAi library for conditional gene inactivation in Drosophila. Nature 448, 151-156 (2007).

121. Frickenhaus, M., Wagner, M., Mallik, M., Catinozzi, M. \& Storkebaum, E. Highly efficient cell-type-specific gene inactivation reveals a key function for the Drosophila FUS homolog cabeza in neurons. Sci Rep 5, 9107, https://doi.org/10.1038/srep09107 (2015).

122. Martin, I., Jones, M. A. \& Grotewiel, M. Manipulation of Sod1 expression ubiquitously, but not in the nervous system or muscle, impacts age-related parameters in Drosophila. FEBS Lett 583, 2308-2314, https://doi.org/10.1016/j.febslet.2009.06.023 (2009).

123. Mizielinska, S. \& Isaacs, A. M. C9orf72 amyotrophic lateral sclerosis and frontotemporal dementia: gain or loss of function? Curr Opin Neurol 27, 515-523, https://doi.org/10.1097/WCO.0000000000000130 (2014).

124. Fromont-Racine, M., Rain, J. C. \& Legrain, P. Building protein-protein networks by two-hybrid mating strategy. Methods in enzymology 350, 513-524 (2002).

125. Guarente, L. Yeast promoters and lacZ fusions designed to study expression of cloned genes in yeast. Methods Enzymol 101, 181-191 (1983).

126. Kushimura, Y. et al. Overexpression of ter94, Drosophila VCP, improves motor neuron degeneration induced by knockdown of TBPH, Drosophila TDP-43. Am J Neurodegener Dis 7, 11-31 (2018).

127. Xia, R. et al. Motor neuron apoptosis and neuromuscular junction perturbation are prominent features in a Drosophila model of Fus-mediated ALS. Mol Neurodegener 7, 10, https://doi.org/10.1186/1750-1326-7-10 (2012).

128. Miguel, L. et al. Accumulation of insoluble forms of FUS protein correlates with toxicity in Drosophila. Neurobiol Aging 33, 1008 e1001-1015, https://doi.org/10.1016/j.neurobiolaging.2011.10.008 (2012).

129. Chen, Y. et al. Expression of human FUS protein in Drosophila leads to progressive neurodegeneration. Protein Cell 2, 477-486, https://doi.org/10.1007/s13238-011-1065-7 (2011).

130. Watson, M. R., Lagow, R. D., Xu, K., Zhang, B. \& Bonini, N. M. A drosophila model for amyotrophic lateral sclerosis reveals motor neuron damage by human SOD1. J Biol Chem 283, 24972-24981, https://doi.org/10.1074/jbc.M804817200 (2008).

131. Zografos, L. et al. Functional characterisation of human synaptic genes expressed in the Drosophila brain. Biol Open 5, 662-667, https://doi.org/10.1242/bio.016261 (2016).

\section{Acknowledgements}

The authors are indebted to Matthew Camilleri for unwavering technical and administrative support. Thanks also goes to Julia Camilleri for assistance with experiments. We are also very grateful to Aaron Voigt, Magalie Lecourtois, Jane Wu, Brian McCabe, and Fabian Feiguin for fly stocks. This work was supported by the University of Malta Research Fund to RJC, and the Malta Council for Science \& Technology Internationalisation Partnership Award to RJC. RC was supported by the Erasmus+ programme of the EU. ML was supported by an Endeavour Scholarship (Malta), part-financed by the EU - European Social Fund under Operational Programme II Cohesion Policy 2014-2020, "Investing in human capital to create more opportunities and promote the wellbeing of society". RMB was supported by a Bjorn Formosa Scholarship for Advanced Research into ALS/MND funded by the non-profit organisation, ALS Malta Foundation, facilitated by the Research Trust (RIDT) of the University of Malta.

\section{Author contributions}

R.J.C. and R.B. conceived and designed the experiments. R.C., M.L., J.C., R.M.B. and R.J.C. performed the experiments and data interpretation. R.J.C. wrote the manuscript with contributions from R.B. and N.V. The authors declare that they have no conflict of interest. 


\section{Competing interests}

The authors declare no competing interests.

\section{Additional information}

Supplementary information is available for this paper at https://doi.org/10.1038/s41598-019-53508-4.

Correspondence and requests for materials should be addressed to R.J.C.

Reprints and permissions information is available at www.nature.com/reprints.

Publisher's note Springer Nature remains neutral with regard to jurisdictional claims in published maps and institutional affiliations.

(c) (i) Open Access This article is licensed under a Creative Commons Attribution 4.0 International License, which permits use, sharing, adaptation, distribution and reproduction in any medium or format, as long as you give appropriate credit to the original author(s) and the source, provide a link to the Creative Commons license, and indicate if changes were made. The images or other third party material in this article are included in the article's Creative Commons license, unless indicated otherwise in a credit line to the material. If material is not included in the article's Creative Commons license and your intended use is not permitted by statutory regulation or exceeds the permitted use, you will need to obtain permission directly from the copyright holder. To view a copy of this license, visit http://creativecommons.org/licenses/by/4.0/.

(C) The Author(s) 2019 